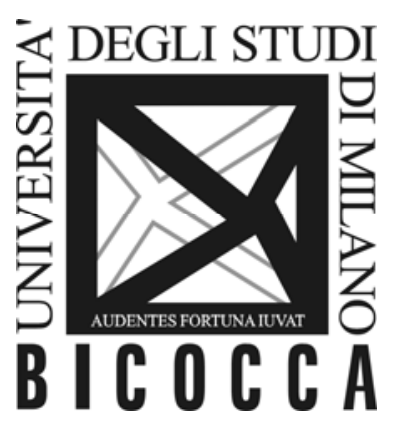

\author{
DEPARTMENT OF ECONOMICS, \\ MANAGEMENT AND STATISTICS \\ UNIVERSITY OF MILAN - BICOCCA
}

DEMS WORKING PAPER SERIES

\title{
Mis-Judging Merit: \\ The Effects of Adjudication Errors in Contests
}

Astrid Gamba, Luca Stanca

No. 345 - July 2016

Dipartimento di Economia, Metodi Quantitativi e Strategie di Impresa Università degli Studi di Milano - Bicocca

http://dems.unimib.it/ 


\title{
Mis-Judging Merit: The Effects of Adjudication Errors in Contests*
}

\author{
Astrid Gamba ${ }^{\dagger}$ Luca Stanca ${ }^{\ddagger}$
}

July 14, 2016

\begin{abstract}
Adjudication errors in contests have a dual nature: they imply at the same time the unjust exclusion of a meritorious candidate (exclusion error) and the unjust inclusion of a non-meritorious one (inclusion error). We study theoretically and experimentally the effects of adjudication errors on contestants' effort, explicitly disentangling the respective effects of exclusion and inclusion errors. We show how behavioral aspects, such as risk aversion, loss aversion and the framing of the incentive scheme (prize vs. penalty) shape the effects of judgement errors on effort. The experimental findings indicate that mis-judgements negatively affect bids, with exclusion and inclusion errors contributing equally to the disincentive effects of adjudication errors. A penalty framing significantly increases bids, relative to a prize framing, both in the absence of judgement errors and in the presence of adjudication errors. On the other hand, no significant interaction is found between the framing of the incentive scheme and the disincentive effects of judgement errors.
\end{abstract}

Keywords: adjudication errors; contests; all-pay auction; experiment JEL classification: C72, C91, D44

${ }^{*}$ We gratefully acknowledge financial support from the Einaudi Institute for Economics and Finance (EIEF). We thank Lorenzo Cappellari, Marco Faravelli and Marco Mantovani for helpful comments. We benefited from participants at the conference Contests: Theory and Evidence, University of East Anglia, and at the IMEBESS conference, LUISS. Any remaining errors are our sole responsability.

${ }^{\dagger}$ Department of Economics, Management and Statistics, University of Milan-Bicocca, Piazza dell'Ateneo Nuovo 1, 20126, Milan, Italy. Corresponding author's email: astrid.gamba@unimib.it, phone: +390264483092.

${ }^{\ddagger}$ Department of Economics Management and Statistics, and Neuro-MI, University of Milan-Bicocca, Piazza dell'Ateneo Nuovo 1, 20126, Milan, Italy. 


\section{Introduction}

The best candidate does not always win. Contests are used to allocate resources in many situations of everyday life (e.g., hirings, promotions, research grants allocations, university admissions, sport tournaments, elections, etc.). They are also used as incentive schemes to extract maximum effort from contestants (e.g., bonus schemes). Even though evaluation committees of such competitions try at their best to abide by fair procedures and objective criteria, misjudgements may occur, preventing a correct identification of the best candidate. As a consequence, adjudication errors produce inefficiency and undermine incentives to exert effort.

Previous research has analyzed errors in contests in different ways. In stochastic contests à la Tullock (1980), originally introduced to describe rent seekers' attempts to condition policy outcomes, success is non-deterministic. The possibility of errors is described by a success function that maps effort levels into probabilities of winning. Different representations of the success function have been proposed in the literature, sharing the common feature that higher relative effort does not necessarily imply success (e.g., Konrad, 2009). While Tullock contests introduce uncertainty in the mapping between effort and success, another strand of the literature, initiated by Lazear and Rosen (1981), represents errors by introducing a wedge between actual and observable effort. In particular, they add some random noise to contestants' effort. This implies that the allocation of prizes is decided on the basis of observable characteristics that misrepresent the effort actually exerted by contestants.

The starting point of our analysis is the consideration that adjudication errors have a dual nature, an important feature that so far has been neglected in the economic literature on contests. Adjudication errors imply at the same time an unjust exclusion, as a deserving candidate is not rewarded (exclusion error), and an unjust inclusion, as an undeserving candidate is rewarded (inclusion error).

In psychology, the duality of errors in contests has been discussed by authors working in the field of behavioral decision making (e.g., Hammond, 1996; Thorngate and Carroll, 1990; Thorngate et al., 2009). However, this strand of research is mostly interested in how evaluation committees make their judgements, and thus focuses on the behavioral aspects and erroneous procedures that may distort their decisions. Less attention is paid to how, in turn, mis-judgements affect the behavior of contestants. Within economics, the duality of errors has been discussed in the literature on crime (e.g., Png, 1986, Kaplow and Shavell, 1994, Polinsky and Shavell, 2009). Rizzolli and Stanca (2012) study experimentally whether the possibility of convicting an innocent (exclusion error) makes deterrence of criminal behavior less effective than the possibility of acquitting a guilty individual (inclusion error).

In non-trivial contests, where the number of contested resources is smaller than the number of competitors, exclusion and inclusion errors generally occur simultaneously, representing two sides of the same coin. However, exclusion and inclusion errors are conceptually distinct. Most importantly, they represent two different channels through which mis-judgements may affect contestants' effort decisions. Un- 
derstanding the role played by exclusion and inclusion errors for effort provision is important for the design of optimal competitive incentive mechanisms. Contest organizers may internalize the fact that adjudication errors can occur and, depending on the economic context, they may place more importance on minimizing the occurrence of either exclusion or inclusion errors. ${ }^{1}$ In fact, while trying to avoid either type of error, they may incur in the other type. They may decide to adopt a permissive (strict) reward scheme in order to avoid excluding (including) the deserving (undeserving) candidate, making in turn exclusion (inclusion) more likely to occur. However, it is not clear which is the lesser evil: is it more disincentivizing to be excessively lenient or strict when rewarding effort?

To answer these questions, we analyze how adjudication errors undermine effort in the strategic context of an all-pay auction (Hillman and Riley, 1989) with two symmetric bidders competing for a monetary prize. We decompose an adjudication error into its exclusion and inclusion components and compare their effects on bids. We start by characterizing the equilibrium bidding behavior in the presence of adjudication errors under the assumption of risk neutrality. We then study how risk aversion and loss aversion influence the effects of adjudication errors. By doing so, we provide different interpretations of the comparative effects of exclusion and inclusion on effort, and provide a better understanding of the disincentive effects of adjudication errors.

For all types of preferences, adjudication errors have a negative impact on effort. While making winning the auction less valuable - due to the possible exclusion they also make losing less unattractive - due to the possible inclusion. Both effects disincentivize effort. In the presence of risk neutral bidders, exclusion and inclusion have the same negative impact on effort, as they reduce its marginal return by the same amount. Risk aversion makes exclusion more detrimental than inclusion: under the assumption that marginal utility is decreasing in monetary outcomes, bidders perceive a larger marginal dis-utility from the exclusion outcome (when the winner does not obtain the reward) relative to the inclusion outcome (when the loser receives the reward).

Loss aversion also exacerbates the negative effects of exclusion relative to inclusion, although the interpretation is different: the loss associated with the erroneous exclusion in case of winning looms larger than the gain associated with the erroneous inclusion in case of losing. However, this result does not apply in the same way under different reward schemes. We show that in a financially equivalent reward structure where, instead of a prize for the winner, a penalties is assigned to loser, ${ }^{2}$ loss aversion produces opposite results and makes the effect of inclusion equal to or stronger than that of exclusion. ${ }^{3}$ In contrast, risk aversion produces the same

\footnotetext{
${ }^{1}$ For example, employers may place higher importance on minimizing the occurrence of inclusion errors rather than exclusion errors as they do not want to waste resources by hiring or promoting the weaker candidate.

${ }^{2}$ Examples of penalties on the workplace are demotion, wage cut, dismissal, unpaid leave of absence, etc.

${ }^{3}$ For a discussion of the comparative effects of positive and negative incentive schemes on team effort see Dickinson (2001).
} 
asymmetric effects in both positive and negative incentive schemes.

We test our theoretical predictions in a laboratory experiment, where we manipulate independently inclusion and exclusion errors, and the framing of the incentive scheme (positive vs. negative), in a $2 \times 2 \times 2$ design. We find that judgement errors negatively affect bids, with exclusion and inclusion errors contributing equally to the disincentive effects of adjudication errors. Interestingly, bids are significantly higher when incentives are framed as penalties, both in the absence of errors and in the presence of adjudication errors. Yet, there is no significant interaction between the framing of the incentive scheme and the disincentive effects of judgement errors.

The paper is organized as follows. Section 2 presents the theoretical framework. Section 3 discusses the experimental design. Section 4 presents the results. Section 5 concludes.

\section{Theoretical Framework}

Consider an all-pay auction with complete information where two symmetric bidders, endowed with $w \geq 0$, simultaneously choose their bids, $b_{1}$ and $b_{2}$, where $b_{i} \in[0, w]$, $i=1,2$, to obtain a monetary prize $\pi \leq w$, which is assigned to the higher bidder (ties are broken randomly).

We introduce the possibility that an adjudication error occurs, so that merit, i.e., the reward, is assigned to the lower and not to the higher bidder. An adjudication error implies two types of error at once: an error of exclusion, whereby merit is denied to the higher bidder - and the reward is assigned to none of the two, and an error of inclusion, whereby merit is attributed to the lower bidder - and the reward is assigned to both. On the basis of this conceptual distinction, we disentangle the two errors (exclusion and inclusion) and analyze how they affect equilibrium bids.

We assume that errors occur with a positive probability $p \in\left(0, \frac{1}{2}\right) .{ }^{4}$ For simplicity, we assume that errors are independent of effort levels.

\subsection{Linear Preferences}

In what follows we describe our baseline model. We assume that bidders are risk neutral, i.e., their Bernoulli utility function of monetary outcomes $u(\cdot)$ is linear and $u(x)=x$. We will relax this assumption in Section 2.2. Propositions 1-4 characterize the Nash equilibrium bidding behavior in every error scenario: no adjudication error (used as a benchmark), exclusion error, inclusion error, and adjudication error (both inclusion and exclusion). Proofs are contained in the Appendix. ${ }^{5}$

\footnotetext{
${ }^{4}$ The assumption that $p<\frac{1}{2}$ is without loss of generality and guarantees that, in every error setting and for every such $p$, there exists a unique equilibrium in mixed strategies.

${ }^{5}$ To prove Proposition 1 we follow the proof of Theorem 1 in Baye et al. (1996) that characterizes the Nash equilibrium of a standard all-pay auction with $n \geq 2$ bidders and homogeneous valuations - and, on the basis of its outline, we build the proofs of propositions 2-4, that describe the equilibrium bidding behavior in the presence of errors.
} 
Proposition 1 (No errors) In the all-pay auction without errors there exists a unique and symmetric Nash equilibrium, such that both players randomize continuously on $[0, \pi]$ according to the $\operatorname{cdf} F^{N}(b)=\frac{b}{\pi}$.

From the cdf we can derive the expected bid as

$$
E^{N}(b)=\int_{0}^{\pi} b d F(b)=\int_{0}^{\pi} \frac{b}{\pi} d b=\frac{\pi}{2}
$$

Next, we consider the setting where an exclusion error may occur (with probability $p$ ). Denote with $q_{i}\left(b_{i}, b_{j}\right)$ the probability that bidder $i$ receives the monetary reward $\pi$, given bids profile $\left(b_{i}, b_{j}\right)$, and define it as

$$
q_{i}\left(b_{i}, b_{j}\right)= \begin{cases}1-p & \text { if } b_{i}>b_{j} \\ \frac{1}{2} & \text { if } b_{i}=b_{j} \\ 0 & \text { if } b_{i}<b_{j}\end{cases}
$$

that is, while the lower bidder never receives the reward, the higher bidder receives the reward only when no exclusion error occurs, i.e., with probability $1-p$. Thus, when the exclusion error occurs (with probability $p$ ), neither bidder receives the reward.

Proposition 2 (Exclusion error) In the all-pay auction with exclusion errors there exists a unique and symmetric Nash equilibrium such that both players randomize continuously on $[0,(1-p) \pi]$ according to the cdf $F^{E}(b)=\frac{b}{(1-p) \pi}$.

From the cdf we derive the expected bid in the presence of exclusion errors as:

$$
E^{E}(b)=\int_{0}^{(1-p) \pi} b d F(b)=\int_{0}^{(1-p) \pi} \frac{b}{(1-p) \pi} d b=\frac{(1-p) \pi}{2} .
$$

Thus, for $p>0$, the average equilibrium bid is lower than in the no-error scenario.

We now examine bidding behavior under inclusion errors. We define the probability of winning of player $i$, given bids profile $\left(b_{i}, b_{j}\right)$, as

$$
q_{i}\left(b_{i}, b_{j}\right)= \begin{cases}1 & \text { if } b_{i}>b_{j} \\ \frac{1}{2} & \text { if } b_{i}=b_{j} \\ p & \text { if } b_{i}<b_{j}\end{cases}
$$

that is, while the higher bidder always receives the reward, when the inclusion error occurs (with probability $p$ ) also the lower bidder receives the reward. Thus, when an inclusion error occurs, both bidders receive the reward.

Proposition 3 (Inclusion error) In the all-pay auction with inclusion errors, there exists a unique and symmetric Nash equilibrium such that both players randomize continuously on $[0,(1-p) \pi]$ according to $F^{I}(b)=\frac{b}{(1-p) \pi}$. 
From the cdf we derive the expected bid as:

$$
E^{I}(b)=\int_{0}^{(1-p) \pi} b d F(b)=\int_{0}^{(1-p) \pi} \frac{b}{(1-p) \pi} d b=\frac{(1-p) \pi}{2} .
$$

Thus, for $p>0$, the average equilibrium bid is lower than in the no-error scenario. Notice that equilibrium bidding behavior in the presence of either exclusion or inclusion errors coincide. That is, exclusion and inclusion produce the same negative effect on effort.

We finally examine equilibrium bidding behavior when an adjudication error occurs with probability $p$. In this case, both exclusion and inclusion are present: the higher bidder does not receive the reward, which is instead assigned to the lower bidder. Thus, we define the probability of receiving the reward for player $i$, given bids profile $\left(b_{i}, b_{j}\right)$, as

$$
q_{i}\left(b_{i}, b_{j}\right)= \begin{cases}1-p & \text { if } b_{i}>b_{j} \\ \frac{1}{2} & \text { if } b_{i}=b_{j} \\ p & \text { if } b_{i}<b_{j}\end{cases}
$$

Proposition 4 (Adjudication error) In the all-pay auction with adjudication errors there exists a unique and symmetric Nash equilibrium such that both players randomize continuously on $[0,(1-2 p) \pi]$ according to $F^{A}(b)=\frac{b}{(1-2 p) \pi}$.

We derive the expected bid in presence of an adjudication error from the equilibrium cdf as

$$
E^{A}(b)=\int_{0}^{(1-2 p) \pi} b d F(b)=\int_{0}^{(1-2 p) \pi} \frac{b}{(1-2 p) \pi} d b=\frac{(1-2 p) \pi}{2} .
$$

Hence, the average equilibrium bid is lower than in the no-error scenario. Notice that, due to linearity,

$$
E^{N}(b)-E^{A}(b)=p \pi=E^{N}(b)-E^{E}(b)+E^{N}(b)-E^{I}(b),
$$

that is, the effect of an adjudication error on expected equilibrium bids is equal to the sum of the effects of its exclusion and inclusion components.

\subsection{Risk Preferences}

In this section, we relax the assumption of linear preferences and analyze the effects of mis-judgements on effort under more general assumptions about risk attitudes.

We first derive the equilibrium behavior of bidders with a generic Bernoulli utility function of monetary outcomes $u(\cdot)$ such that $u^{\prime}(x) \geq 0$ and $u(0)=0$. Then, we analyze how the effects of adjudication errors depend on assumptions about the second derivative of $u(\cdot)$. Since we cannot derive explicit solutions starting from a generic $u(\cdot)$, we compare qualitatively the equilibrium behavior in the different error scenarios by studying the equilibrium cdfs and their supports. Notice that by keeping the assumption that utility is increasing in monetary outcomes, the 
basic trade-off of standard all-pay auctions prevails even in the presence of errors: increasing the bid increases the probability of winning but decreases the monetary payoff.

Consider the setting without errors. The equilibrium cdf, that we call $F^{N}(b)$, satisfies the indifference condition:

$$
F^{N}(b) u(w+\pi-b)+\left[1-F^{N}(b)\right] u(w-b)=u(w)
$$

where the left hand side represents the expected utility from any bid $b$, that depends on whether $b$ is the highest bid or not, while the right hand side is the expected utility from bidding zero (i.e., the opportunity cost of the bid). By rearranging terms, we obtain that the equilibrium cdf is equal to:

$$
F^{N}(b)=\frac{u(w)-u(w-b)}{u(w+\pi-b)-u(w-b)} .
$$

It is immediate to show that bidders randomize continuously on the interval $[0, \pi]$ according to $F^{N}(\cdot) .^{6}$

Consider now the setting with adjudication errors, where the reward might be assigned to the lower rather than to the higher bidder. The equilibrium cdf, that we call $F^{A}(b)$, satisfies the indifference condition:

$$
\begin{gathered}
F^{A}(b)[(1-p) u(w+\pi-b)+p u(w-b)]+\left[1-F^{A}(b)\right][p u(w+\pi-b)+(1-p) u(w-b)]= \\
p u(w+\pi)+(1-p) u(w)
\end{gathered}
$$

where the left hand side is the expected utility from any positive bid, that depends on whether it is the highest bid and on the probability that an error occurs $(p)$, which may affect the expected utility both in case of winning and in case of losing. The right hand side is the expected utility from bidding zero (and losing), which is affected by the positive probability of receiving the reward (due to inclusion). By re-arranging terms, we obtain that the cdf that solves the equilibrium indifference condition (2) is

$$
F^{A}(b)=\frac{(1-p)[u(w)-u(w-b)]+p[u(w+\pi)-u(w+\pi-b)]}{(1-2 p)[u(w+\pi-b)-u(w-b)]} .
$$

It is immediate to show that in equilibrium bidders randomize continuously on the support $\left[0, \bar{b}^{A}\right]$ according to $F^{A}(\cdot)$, where $\bar{b}^{A}$ is the solution to condition (2), given $F^{A}\left(\bar{b}^{A}\right)=1 .^{7}$

\footnotetext{
${ }^{6}$ Recall that the upper bound of the support of $F^{N}(\cdot)$ can be found by imposing that the expected utility from bidding exactly the upper bound (and winning) is equal to the expected utility from bidding zero. It is immediate to verify that $\pi$ solves $u(w+\pi-b)=$ $u(w)$ (i.e., equation $(1)$ with $F^{N}(\pi)=1$ ). The rest of the proof follows the proof of Proposition 1 in Appendix A.

${ }^{7}$ The complete proof that this is the equilibrium strategy profile essentially replicates the proof of Proposition 4 contained in Appendix A, except for the equilibrium indifference condition that delivers $F^{A}(\cdot)$ and the characterization of the upper bound of the support.
} 
To analyze the effect of adjudication errors on effort, we perform a qualitative analysis as follows. First, we compare the two equilibrium cdfs. It can be shown that the distribution of bids in the no error scenario first order stochastically dominates the distribution of bids in the adjudication error scenario, i.e., for every $b$ in a generic support $[0, \bar{b}]$, it holds that $F^{N}(b) \leq F^{A}(b)$. Second, we compare the supports of the two cdfs. In particular, we argue that $\bar{b}^{A}$ is smaller than $\pi$. This can be verified by evaluating condition (2) for $b=\pi$. Indeed, the expected utility from winning the auction by bidding exactly $\pi$ in the presence of an adjudication error (left hand side of condition (2) with $F^{A}(\pi)=1$ ) is smaller than the expected utility from bidding zero (right hand side of condition (2)). Thus, we obtain the following relation between expected bids in the two settings:

$$
E^{A}(b)=\int_{0}^{\bar{b}^{A}}\left[1-F^{A}(b)\right] d b \leq \int_{0}^{\pi}\left[1-F^{A}(b)\right] d b \leq \int_{0}^{\pi}\left[1-F^{N}(b)\right] d b=E^{N}(b) .
$$

Hence, the presence of adjudication errors decreases average bids for every $u(\cdot)$ increasing in its argument.

Consider now the exclusion and the inclusion error settings, where either error occurs with probability $p$. We examine first the exclusion error scenario, where the highest bidder may not receive the reward. The equilibrium cdf $F^{E}(b)$ for an individual with utility function $u(\cdot)$ must satisfy the indifference condition:

$$
F^{E}(b)[(1-p) u(w+\pi-b)+p u(w-b)]+\left[1-F^{E}(b)\right] u(w-b)=u(w)
$$

from which we obtain that

$$
F^{E}(b)=\frac{u(w)-u(w-b)}{(1-p)[u(w+\pi-b)-u(w-b)]} .
$$

Consider the inclusion error setting, where the lowest bidder may receive the reward. The equilibrium cdf $F^{I}(b)$ must satisfy the indifference condition:

$$
\begin{aligned}
F^{I}(b) u(w+\pi-b)+\left[1-F^{I}(b)\right][(1-p) u(w-b)+p u(w+\pi-b)] & = \\
(1-p) u(w) & +p u(w+\pi)
\end{aligned}
$$

so that

$$
F^{I}(b)=\frac{(1-p)[u(w)-u(w-b)]+p[u(w+\pi)-u(w+\pi-b)]}{(1-p)[u(w+\pi-b)-u(w-b)]} .
$$

In order to establish a univocal relation between expected bids in the two error settings we need to make further assumptions about the shape of $u(\cdot)$. Thus, we assume that $u(\cdot)$ is concave and compare expected bids in the presence of the two types of errors when bidders are risk averse. 
Due to decreasing marginal utility, the marginal dis-utility from the exclusion outcome $\left(u^{\prime}(w-b)\right)$ - i.e., the winner does not receive the reward - is larger than the marginal dis-utility from the inclusion outcome $\left(u^{\prime}(w+\pi-b)\right)$ - i.e., the loser receives the reward. In other words, the exclusion error raises the cost of bidding more than the inclusion error with respect to the benchmark. Thus, for risk averse bidders, exclusion generates a stronger distortion, relative to inclusion, on the incentives to exert effort. We can state the following proposition (the proof is in Appendix A).

Proposition 5 When bidders are risk averse, exclusion errors have a stronger negative effect on average equilibrium bids than inclusion errors.

The opposite result holds for risk-loving bidders, as their marginal utility is increasing in monetary outcomes.

To sum up, by comparing all cdfs it is easy to show that with generic risk preferences $F^{A}(b) \geq F^{X}(b) \geq F^{N}(b)$, with $X=E, I$ (with strict inequality for $b>0$ ). Their supports display the same lower bound of zero and upper bounds that satisfy: $\bar{b}^{A} \leq \bar{b}^{X} \leq \bar{b}^{N}$, with $X=E, I{ }^{8}$ Hence, it must be the case that $E^{N}(b) \leq$ $E^{X}(b) \leq E^{A}(b)$. Yet, the relations between $F^{E}(b)$ and $F^{I}(b)$ and between $\bar{b}^{E}$ and $\bar{b}^{I}$ depend on the curvature of $u(\cdot)$. When bidders are risk averse, $F^{E}(b)>F^{I}(b)$ (for every $b$ ) and $\bar{b}^{E} \leq \bar{b}^{I}$ so that $E^{E}(b) \leq E^{I}(b)$, while the opposite relations hold true when bidders are risk lovers.

\subsection{Loss Aversion}

In this section we analyze the effects of adjudication errors on effort when bidders are loss averse. Specifically, we assume that the utility function of monetary outcomes satisfies $u(x)=x$ for $x \geq 0$ and $u(x)=-\lambda u(-x)$ for $x<0$, with $\lambda>1$. We also assume that bidders consider their endowment $w$ as a reference point and evaluate monetary outcomes as positive or negative variations with respect to $w$.

Loss aversion can be expected to have a negative effect on effort, as it exacerbates its cost in case of losing. In what follows we describe how loss aversion influences the effects of judgement errors on effort. In order to do so, it is useful to introduce an alternative reward structure, whereby incentives are framed as losses instead of gains. We call the reward structure based on monetary gains a prize scheme, and the alternative reward structure based on monetary losses a penalty scheme. The penalty scheme is such that the lowest bidder receives a monetary penalty and the highest bidder does not. Notice that, the two schemes provide contestants with the same incentives to exert effort as they both award merit to the highest bidder. Yet, rewards are different: instead of obtaining a prize, in a penalty scheme, the highest

\footnotetext{
${ }^{8}$ To see that $\bar{b}^{A}<\bar{b}^{E}$ it is enough to check that bidders are better off by bidding zero instead of $\bar{b}^{E}$ in the adjudication error scenario, i.e., $(1-p) u\left(w+\pi-\bar{b}^{E}\right)+p u\left(w-\bar{b}^{E}\right)<$ $p u(w+\pi)+(1-p) u(w)$, which is always true given that $\bar{b}^{E}$ satisfies condition (3). Similarly, it is immediate to see that $\bar{b}^{I}<\bar{b}^{N}=\pi$ as bidders are clearly better off by bidding zero rather than $\pi$ in an inclusion error scenario. Indeed, $u(w+\pi-\pi)<p u(w+\pi)+(1-p) u(w)$.
} 
bidder avoids a penalty. When an adjudication error occurs, the penalty is assigned to the highest and not to the lowest bidder. An exclusion error implies that (also) the highest bidder unjustly receives the penalty, while an inclusion error implies that neither bidder receives it.

In order to make the penalty and the prize scheme financially equivalent, we assume that in the penalty scheme bidders are endowed with $2 w$, and that the penalty is as large as the prize $\pi$. Thus, monetary outcomes in every error scenario are algebraically identical across the two schemes. ${ }^{9}$ Consider, for instance, the scenario without errors and call $G^{N}(\cdot)$ the penalty scheme counterpart of $F^{N}(\cdot)$ derived from condition (1). In a penalty scheme the equilibrium indifference condition for bidders endowed with a generic $u(\cdot)$ becomes

$$
G^{N}(b) u(2 w-b)+\left[1-G^{N}(b)\right] u(2 w-\pi-b)=u(2 w-\pi) .
$$

It is immediate to see that conditions (1) and (5) coincide, due to payoff equivalence, and that this equivalence relation applies to every error scenario. This implies that all the results presented in propositions 1-5 also hold in a penalty scheme.

However, under loss aversion, bidding behavior across the two schemes may differ. Every outcome in any error scenario of a penalty scheme is such that bidders bear at best the bid and at worst both the penalty and the bid. Thus, under loss aversion, condition (5) becomes:

$$
G^{N}(b)[-\lambda u(b)]+\left[1-G^{N}(b)\right][-\lambda u(\pi+b)]=-\lambda u(\pi)
$$

Since all outcomes are in the loss domain, loss aversion does not have any bite in a penalty scheme and

$$
G^{N}(b)=\frac{u(\pi+b)-u(\pi)}{u(\pi+b)-u(b)} .
$$

In order to measure the impact of loss aversion on the disincentive effects of errors, we assume for simplicity that $u(x)=x$ for $x \geq 0$ and $u(x)=-\lambda x$ for $x<0$. With linear preferences, in every error setting, the average equilibrium bid of a penalty scheme coincides with the average equilibrium bid of the baseline model of Section 2.1 (prize scheme with standard linear preferences). Hence, it is equivalent to compare the predictions for a prize scheme with loss averse bidders with either the predictions for penalty scheme with loss averse bidders or those of the simple setting of Section 2.1. In the presentation of the following results we adopt the prize versus penalty comparison, as it makes it easier to interpret the role of loss aversion in shaping the disincentive effects of errors on bids.

\footnotetext{
${ }^{9}$ Notice that size of the endowment does not have any implications under the reference dependence assumption. Yet, reference dependence is a specific assumption on the bidders' subjective utility, while we want that our set up may ideally suit alternative interpretations. Thus, we construct the two payoff structures in a way that makes the two schemes payoffequivalent and, thus, comparable even without any specific superstructure on the bidders' preferences.
} 
We first analyze the effect of loss aversion on expected bids in the benchmark without errors. We find that loss averse contestants bid less aggressively in a prize than in a penalty scheme, as stated in the following proposition.

Proposition 6 In a scenario without errors with loss averse bidders, bids in a penalty scheme are higher than in a prize scheme.

Proof. The equilibrium mixed strategy profile in this setting can be derived by replicating the steps of the proof of Proposition 1 (see Appendix), except for point 4. Any bid in the support should provide the same expected utility from bidding zero, that is

$$
F^{N}(b)(\pi-b)+\left[1-F^{N}(b)\right](-\lambda b)=0 .
$$

The indifference condition yields

$$
F^{N}(b)=\frac{\lambda b}{\pi+(\lambda-1) b} .
$$

The support of the equilibrium cdf does not change with respect to the benchmark and coincides with $[0, \pi]{ }^{10}$ Thus, we can derive the average equilibrium bid of loss averse bidders as

$$
\int_{0}^{\pi} \frac{\lambda \pi}{(\pi+b(\lambda-1))^{2}} d b=\frac{1-\lambda(1-\log \lambda)}{(\lambda-1)^{2}} \pi .
$$

that, for every $\lambda>1$, is smaller than $\frac{\pi}{2}$, which is the average equilibrium bid in the benchmark, and it decreases with $\lambda$.

The intuition for this result is that loss averse bidders are more averse to the loss that derives from losing in a penalty scheme than they are attracted to the gain that derives from winning in a prize scheme. This result is in line with recent evidence showing that loss contracts increase individual and team performance in the workplace (see Section 5 for a discussion). Importantly, our result suggests that this may occur also in a competitive environment: negative incentive schemes may be more effective to extract effort as, due to loss aversion, contestants are more willing to exert effort in order to avoid losses.

Next, we analyze how loss aversion influences the effects of judgement errors on average bids by comparing equilibrium behavior across prize and penalty schemes, under exclusion and inclusion errors, respectively.

Proposition 7 Under loss aversion, for $\lambda$ large enough, exclusion errors have a stronger negative effect on bids in a penalty scheme than in a prize scheme.

Proof. The equilibrium mixed strategy profile in this setting can be derived by replicating the steps of the proof of Proposition 2, except for points $2 \mathrm{~b}$ and 4 . Any bid in the support must provide the same expected utility from bidding zero that is

\footnotetext{
${ }^{10} \mathrm{It}$ is immediate to verify that $\pi$ satisfies point $2 . \mathrm{b}$ of the proof of Proposition 1.
} 


$$
F^{E}(b)[(1-p)(\pi-b)+p(-\lambda b)]+\left[1-F^{E}(b)\right](-\lambda b)=0 .
$$

This indifference condition yields

$$
F^{E}(b)=\frac{\lambda b}{(1-p)[\pi+(\lambda-1) b]} .
$$

In addition, the support of the equilibrium mixed strategy now differs with respect to the one of the exclusion error scenario of the baseline model and it is equal to $\bar{b}=\frac{(1-p) \pi}{1+p(\lambda-1)}$, that solves $(1-p)(\pi-b)+p(-\lambda b)=0$, that corresponds to condition (7) with $F^{E}(\bar{b})=1$. Thus, we can derive the average equilibrium bid of loss averse bidders as

$$
\int_{0}^{\bar{b}} b \frac{\lambda \pi}{(1-p)[\pi+b(\lambda-1)]^{2}} d b
$$

The disincentive effect of an exclusion error with loss averse bidders in a prize scheme is smaller relative to a penalty scheme if the following inequality holds true:

$$
\begin{aligned}
\frac{1-\lambda(1-\log \lambda)}{(\lambda-1)^{2}} \pi-\frac{(\lambda-1)(p-1)+\lambda \log \lambda-\lambda \log [1+(\lambda-1) p]}{(\lambda-1)^{2}(p-1)} & \\
& <\frac{\pi}{2}-\frac{\pi(1-p)}{2}
\end{aligned}
$$

where the left hand side is the difference between the average bid in the no error scenario and the average bid in an exclusion error scenario of a prize scheme; the right hand side measures the effect of an exclusion error in a penalty scheme. It can be shown that this inequality is not satisfied for every $(\lambda, p)$, such that $\lambda>1$ and $p \in\left(0, \frac{1}{2}\right)$. Let us denote $\bar{\lambda}$ the degree of loss aversion above which the inequality is satisfied as a function of $p$, i.e., $\bar{\lambda}=f(p)$. Let us divide both sides for $\pi$ and denote the resulting expression on the left hand side as $D(\lambda, p)$. The above inequality becomes

$$
D(\lambda, p)=\frac{1-\lambda(1-\log \lambda)}{(\lambda-1)^{2}}-\frac{(\lambda-1)(p-1)+\lambda \log \lambda-\lambda \log [1+(\lambda-1) p]}{(\lambda-1)^{2}(p-1)}<\frac{p}{2} .
$$

and $\bar{\lambda}$ solves $D(\bar{\lambda}, p)-\frac{p}{2}=0$. It can be shown that $\bar{\lambda}$ decreases with $p$. That is, as the probability of an error grows large, less loss aversion is needed to make an exclusion error in a prize scheme at least as detrimental as in a penalty scheme.

Proposition 8 Under loss aversion, inclusion errors have a stronger negative effect on bids in a penalty scheme than in a prize scheme.

Proof. The equilibrium mixed strategy profile in this setting can be derived by replicating the steps of the proof of Proposition 3, except for point 4. Any bid in the support must provide the same expected utility from bidding zero that is

$$
F^{I}(b)(\pi-b)+\left[1-F^{I}(b)\right][(1-p)(-\lambda b)+p(\pi-b)]=p \pi .
$$


This indifference condition yields

$$
F^{I}(b)=\frac{[(1-p) \lambda+p] b}{(1-p)[\pi+b(\lambda-1)]} .
$$

The support of the equilibrium cdf is the same as in the inclusion error scenario of the baseline model and it is equal to $\bar{b}=(1-p) \pi .{ }^{11}$ Thus, we can derive the average equilibrium bid of loss averse bidders as

$$
\int_{0}^{\bar{b}} b \frac{\pi[\lambda(1-p)+p]}{[\pi+b(\lambda-1)]^{2}(1-p)} d b .
$$

The disincentive effect of an inclusion error with loss averse bidders in a prize scheme is smaller relative to a penalty scheme if the following inequality holds:

$$
\begin{aligned}
& \frac{1-\lambda(1-\log \lambda)}{(\lambda-1)^{2}} \pi-\frac{(\lambda-1)(1-p)+[(\lambda-1) p-\lambda] \log [1-(\lambda-1) p]}{(\lambda-1)^{2}(p-1)} \pi \\
&<\frac{\pi}{2}-\frac{\pi(1-p)}{2}
\end{aligned}
$$

where the left hand side is the difference between the average bid in the no error scenario and the average bid of an inclusion error scenario in a prize scheme; the right hand side measures the effect of an inclusion error in a penalty scheme. It can be shown that this inequality is satisfied for every $(\lambda, p)$, such that $\lambda>1$ and $p \in\left(0, \frac{1}{2}\right)$.

In the presence of exclusion errors, loss-averse contestants perceive a negative variation in case of winning that is smaller in a prize than in a penalty scheme, as the loss looms larger than the non-gain. Similarly, in the presence of inclusion errors, loss-averse contestants perceive a positive variation in case of losing that is larger in a penalty scheme than in a prize scheme, as the avoided loss looms larger than the prize. However, inclusion, differently from exclusion, has an additional disincentive effect. Specifically, it affects the expected payoff from exerting zero effort, as in some contingencies the bidder receives the prize (prize scheme) or avoids the penalty (penalty scheme) at no cost. Since the non-penalty looms larger than the prize, inclusion induces a variation in the opportunity cost of effort that is asymmetric across the two reward structures: the opportunity cost increases more in a penalty scheme than in a prize scheme. Overall, these two effects of inclusion induce a disincentive effect that is unambiguously larger in a penalty relative to a prize scheme. $^{12}$

\footnotetext{
${ }^{11}$ Obviously, the upper bound of the support does not change with respect to the baseline model since the inclusion error does not affect the expected payoff from winning.

${ }^{12}$ Notice that exclusion does not have any effect on the bid's opportunity cost. Exclusion has only a negative effect on the expected payoff from winning the auction that is larger in a penalty than in a prize scheme. However, this effect may be not sufficient to generate an asymmetry across the two schemes, when the degree of loss aversion is relatively low.
} 
We now analyze how the effect of an adjudication error varies across the two reward schemes. By comparing the difference between the benchmark average equilibrium bid and the average equilibrium bid in the presence of adjudication errors, across the prize and the penalty scheme, we obtain the following result:

Proposition 9 Under loss aversion, adjudication errors have a stronger negative effect on bids in a penalty than in a prize scheme.

Proof. In a penalty scheme loss averse bidders behave like in the baseline model and randomize continuously on the support $[0,(1-2 p) \pi]$ according to the cdf:

$$
G^{A}(b)=\frac{b}{(1-2 p) \pi}
$$

so that the average equilibrium bid is $\frac{(1-2 p) \pi}{2}$. Thus, the difference between average equilibrium bids in the penalty scheme without and with adjudication errors can be computed as

$$
\frac{\pi}{2}-\frac{(1-2 p) \pi}{2}=p \pi
$$

In contrast, due to loss aversion, the equilibrium cdf in a prize scheme differs with respect to the benchmark as it must satisfy the indifference condition

$$
F^{A}(b)[(1-p)(\pi-b)+p(-\lambda b)]+\left[1-F^{A}(b)\right][(1-p)(-\lambda b)+p(\pi-b)]=p \pi
$$

that yields

$$
F^{A}(b)=\frac{[\lambda-p(\lambda-1)] b}{(1-2 p)[\pi+(\lambda-1) b]}
$$

where the upper bound of the support is now $\bar{b}=\frac{(1-2 p) \pi}{1+p(\lambda-1)} \cdot{ }^{13}$ The proof that this is indeed the equilibrium mixed strategy of every bidder follows the proof of Proposition 4.

The disincentive effect of an adjudication error with loss averse bidders in a prize scheme is computed as:

$$
\pi \frac{[1-\lambda(1-\log \lambda)]}{(\lambda-1)^{2}}-\int_{0}^{\bar{b}} b \frac{\pi(\lambda-(\lambda-1) p)}{(1-2 p)(\pi+b(\lambda-1))^{2}} d b
$$

where the first term is the average equilibrium bid in the no error scenario (derived from the proof of Proposition 6) and the second term is the average equilibrium bid in the adjudication error scenario. We can rewrite the last expression as

\footnotetext{
${ }^{13}$ The upper bound of the support is derived from the condition that expected utility from the equilibrium strategy in the best case scenario (left hand side of condition (8) with $\left.F^{A}(b)=1\right)$ must be equal to the expected utility from bidding zero.
} 


$$
\begin{aligned}
\pi & {\left[\frac{1-\lambda(1-\log \lambda)}{(\lambda-1)^{2}}+\right.} \\
& \left.-\frac{(\lambda-1)(1-2 p)+(\lambda-p(\lambda-1))\{\log [1+(\lambda-1) p]-\log [\lambda-p(\lambda-1)]\}}{(\lambda-1)^{2}(2 p-1)}\right] .
\end{aligned}
$$

It can be shown that this expression is (strictly) smaller than $p \pi$ for every $\lambda>1$.

To summarize, when bidders are loss averse, average bids are lower in a prize scheme than in a penalty scheme, but the negative effect of adjudication errors on incentives is less strong in a prize scheme. ${ }^{14}$ These results have interesting implications for the design of optimal contests. Under loss aversion, penalty schemes may better suit the objective of contest designers to maximize effort insofar as adjudication errors are absent. When instead adjudication schemes are defective and errors may occur, positive incentives has to be preferred to negative incentives.

Next, we consider how loss aversion provides an alternative interpretation to risk aversion for why exclusion may have a stronger disincentive effect than inclusion. The intuition is that when bidders evaluate monetary outcomes as variations (namely, gains or losses) relative to their endowment, exclusion generates a possible loss in case of winning, while inclusion generates a possible gain in case of losing. First, we analyze the effect of loss aversion on the relation between the two disincentive effects in a prize scheme and in a penalty scheme respectively. We can now develop the analysis at a more general level and study the relation between exclusion and inclusion with weaker assumptions on preferences, i.e., not necessarily linear.

Under loss aversion, condition (3), i.e., the equilibrium cdf under exclusion errors, becomes:

$$
F^{E}(b)=\frac{u(0)-u(-b)}{(1-p)[u(\pi-b)-u(-b)]}=\frac{\lambda u(b)}{(1-p)[u(\pi-b)+\lambda u(b)]} .
$$

Under loss aversion, condition (4), i.e., the equilibrium cdf under inclusion errors, becomes:

$$
\begin{aligned}
F^{I}(b)=\frac{(1-p)[u(0)-u(-b)]+p[u(\pi)-u(\pi-b)]}{(1-p)[u(\pi-b)-u(-b)]} & = \\
& \frac{(1-p) \lambda u(b)+p[u(\pi)-u(\pi-b)]}{(1-p)[u(\pi-b)+\lambda u(b)]} .
\end{aligned}
$$

For loss averse bidders, the possible loss due to exclusion looms larger than the possible gain due to inclusion. Thus, exclusion gives rise to a harsher distortion of the incentive to exert effort with respect to inclusion, as bidders prefer avoiding the loss when winning to making the gain when losing. This intuition leads to the following proposition (the proof is in Appendix A).

\footnotetext{
${ }^{14} \mathrm{An}$ alternative way to state these results is that in the absence of errors loss aversion decreases average bids and it decreases the disincentive effect of an adjudication error.
} 
Proposition 10 In a prize scheme with loss averse bidders, exclusion errors have a stronger negative effect on bids than inclusion errors.

We now analyze how the effects of the two components of an adjudication error - exclusion and inclusion - affect effort in a penalty scheme. The equilibrium cdf under exclusion errors in a penalty scheme, for a generic $u(x)$, satisfies:

$G^{E}(b)[(1-p) u(2 w-b)+p u(2 w-\pi-b)]+\left[1-G^{E}(b)\right] u(2 w-\pi-b)=u(2 w-\pi)$.

Thus, for loss averse bidders, it is equal to:

$$
G^{E}(b)=\frac{u(\pi+b)-u(\pi)}{(1-p)[u(\pi+b)-u(b)]}
$$

with support $\left[0, \bar{b}^{E}\right]$, whereby $\bar{b}^{E}$ solves $(1-p) u(b)+p u(\pi+b)=u(\pi)$.

The equilibrium cdf under inclusion errors in a penalty scheme, for a generic $u(x)$, satisfies:

$G^{I}(b) u(2 w-b)+\left[1-G^{I}(b)\right][(1-p) u(2 w-\pi-b)+p u(2 w-b)]=(1-p) u(2 w-\pi)+p u(2 w)$

Thus, for loss averse bidders it is equal to:

$$
G^{I}(b)=\frac{(1-p)[u(\pi+b)-u(\pi)]+p u(b)}{(1-p)[u(\pi+b)-u(b)]}
$$

with support $\left[0, \bar{b}^{I}\right]$, whereby $\bar{b}^{I}$ solves $u(b)=(1-p) u(\pi)$.

By comparing the two equilibrium cdfs, conditions (9) and (10), respectively, we obtain the following proposition. The proof is contained in Appendix A.

Proposition 11 In a penalty scheme with loss averse bidders, inclusion errors have stronger negative effect on bids than exclusion errors. In particular:

Thus, similarly to risk aversion, loss aversion generates an asymmetry between the negative effects of exclusion and inclusion on average bids. However, the effect of loss aversion strongly depends on how the incentive scheme is framed and it does not characterize defective contests in general. In particular, it does not apply to environments where contest designers adopt penalty schemes to incentivize effort.

The results of propositions 5, 10 and 11 can be summarized as follows: with positive incentive schemes, both risk aversion and loss aversion imply that exclusion errors are more detrimental for effort than inclusion errors. In contrast, with negative incentive schemes, while the implications of risk aversion do not change, loss aversion has an opposite effect: exclusion has a less strong disincentive effect than inclusion. 


\section{The Experiment}

The baseline experimental task is a standard all-pay auction where two subjects compete for a monetary reward. Subjects are matched in pairs and have a monetary endowment that they can spend to buy tickets at the cost of 1 ECU per ticket. The subject who buys most tickets wins the contest (ties are broken randomly). However, the winner of the contest may not obtain the reward, depending on the occurrence of a random mis-judgement error, as detailed below.

\subsection{Design}

We manipulate exogenously the presence of exclusion and inclusion errors, and the framing of the incentive scheme (prize vs penalty), in a $2 \times 2 \times 2$ factorial design. In all treatments, the highest bidder wins the contest. However, the identification of the winner (loser) of the contest may be subject to either exclusion or exclusion errors, or both. ${ }^{15}$ In T1, no errors occur, so that the winner obtains the reward with certainty. In T2, an error of exclusion may occur with probability $\frac{1}{4}$. In T3, an error of inclusion may occur with probability $\frac{1}{4}$. In T4, an adjudication error may occur with probability $\frac{1}{4}$, implying both exclusion and inclusion at the same time.

To summarize, we implement the following four treatments:

T1 - No errors: the winner obtains the reward, while the loser does not obtain it;

T2 - Exclusion error:

- with probability $\frac{1}{4}$ the winner does not obtain the reward (thus no bidder obtains the reward);

- with probability $\frac{3}{4}$ the winner obtains the reward while the loser does not obtain it;

\section{T3 - Inclusion error:}

- with probability $\frac{1}{4}$ the loser obtains the reward (thus both bidders obtain the reward);

- with probability $\frac{3}{4}$ the winner obtains the reward while the loser does not obtain it;

\section{T4 - Inclusion and Exclusion (Adjudication) error:}

- with probability $\frac{1}{4}$ the reward is assigned to the loser and not to the winner;

- with probability $\frac{3}{4}$ the winner obtains the reward while the loser does not obtain it;

\footnotetext{
${ }^{15}$ The occurrence of an error is determined by a coin toss. Subjects are informed about the coin toss realization after their bidding decision.
} 
Each of these four treatments is implemented under two different framings, with the reward consisting of either receiving a monetary prize (Prize framing) or avoiding a monetary penalty (Penalty framing), respectively. In the Prize framing, subjects are endowed with 1000 ECUs and the reward is a prize of 1000 ECU. For subjects who are awarded the prize, the payoff is given by the endowment, minus the cost of the tickets, plus the prize; for subjects who are not awarded the prize, the payoff is the endowment minus the cost of the tickets. In the Penalty framing, subjects are endowed with 2000 ECUs and compete to avoid a penalty of 1000 ECUs. For subjects who receive the penalty the payoff is the endowment minus the cost of the tickets minus the penalty; for subjects who do not receive the penalty the payoff is the endowment minus the cost of the tickets. The four treatments are therefore payoff-equivalent across the two framings. Table 1 summarizes the features of all treatments.

Table 1: Experimental Treatments

\begin{tabular}{lcccc}
\hline & \multicolumn{2}{c}{ Prize framing } & \multicolumn{2}{c}{ Penalty framing } \\
& Win & Lose & Win & Lose \\
\hline T1 - No errors & Prize & No prize & No penalty & Penalty \\
T2 - Exclusion error & No prize & No prize & Penalty & Penalty \\
T3 - Inclusion error & Prize & Prize & No penalty & No penalty \\
T4 - Exclusion and Inclusion & No prize & Prize & Penalty & No penalty \\
\hline
\end{tabular}

\subsection{Hypotheses}

Under standard risk-neutral preferences, Nash equilibrium strategy profiles and expected bids for each treatment can be summarized as follows. In T1, both players randomize continuously on $[0,1000]$ according to the cdf $F(b)=b$, with an expected bid of 500. In T2 and T3, both players randomize continuously on $\left[0, \frac{3}{4}\right]$ according to $F(b)=\frac{4}{3} b$, with an expected bid of 375 . In T4, both players randomize continuously on $\left[0, \frac{1}{2}\right]$ according to $F(b)=2 b$, with an expected bid of 250. Table 2 compares expected individual bids in ECUs for each of the four treatments.

Table 2: Expected bids, by treatment

\begin{tabular}{lccc}
\hline & & \multicolumn{2}{c}{ Inclusion error } \\
& Winner rewarded & 500 & 375 \\
Exclusion error & & & \\
& & 375 & 250 \\
\hline
\end{tabular}

The first set of hypotheses is about the effects of judgement errors on bids in the absence of reference-dependent preferences (i.e., abstracting from the framing of the task). Adjudication errors are expected to have a negative effect on bids under any 
expected-utility preferences. This reflects two complementary effects: the exclusion error component decreases the expected utility of a winning bid; the inclusion error component increases the expected utility of a losing bid, thus decreasing the expected net returns from bidding. In addition, under linear utility no interaction is expected between exclusion and inclusion errors, so that the effects of adjudication errors are equal to the sum of exclusion and inclusion errors (additivity). Alternatively, under non-linear utility, exclusion and inclusion errors may be complement or substitutes.

If utility is linear in monetary outcomes, exclusion and inclusion errors have the same negative impact on the marginal return from bidding and, therefore, on expected bids. On the other hand, if utility is non-linear, the two errors have different effects on bids. Under risk aversion, the expected utility from bidding, both in case of winning and in case of losing, is lower in the presence of exclusion than inclusion errors. ${ }^{16}$ Therefore, for a given error probability, risk averse individuals are expected to bid less in the presence of errors of exclusion than of inclusion. Hence, whereas under linear utility exclusion and inclusion errors have the same disincentive effect, under risk aversion exclusion errors have a stronger disincentive effect on bids than inclusion errors.

Summing up, we test the following hypotheses about the effects of judgement errors on effort:

H1a: Adjudication errors have a negative effect on bids.

H1b: Both exclusion and inclusion errors have a negative effect on bids.

H1c: Exclusion errors have a stronger disincentive effect on bids than inclusion errors.

H1d: The effects of exclusion and inclusion errors are non-additive.

The second set of hypotheses concerns the effects of the framing of the incentive scheme, and the interaction with judgement errors, focusing on the role played by loss aversion. Under expected-utility preferences, average bids are equal in the Prize and Penalty framings, irrespective of risk attitudes, since the monetary payoffs are exactly the same in the two settings. On the other hand, loss aversion would produce different bids, and different effects of judgement errors, in the two framings.

In the absence of errors, loss aversion implies that bids are higher in a penalty framing, since the loss perceived in case of losing in a penalty scheme weights more than a corresponding non-gain in a prize scheme. Hence bidders want to avoid losing in Penalty more than they want to avoid it in Prize. ${ }^{17}$ More importantly, under loss

\footnotetext{
${ }^{16}$ The reason for this result is that in case of winning with probability $\left(\frac{3}{4}\right)$ monetary outcomes are the same in $\mathrm{T} 2$ and $\mathrm{T} 3$, but with probability $\left(\frac{1}{4}\right)$ the winner does not receive the reward and the payoff is lower in T3 than in T2. In case of losing, with probability $\left(\frac{3}{4}\right)$ the payoff in T2 and in T3 is the same, but with probality $\left(\frac{1}{4}\right)$ the loser receives the reward, so that the payoff is higher in T3 than T2.

${ }^{17}$ Note that reflection may have a similar effect, depending on the curvature of the utility function.
} 
aversion adjudication errors have a stronger disincentive effect on bids in the Penalty than in the Prize framing (see Proposition 9). The intuition is the following: in a Penalty framing, loss averse subjects perceive a larger negative (positive) variation in the expected utility from winning (losing) with respect to the benchmark than in a Prize framing. Thus, the disincentive effect of adjudication errors is stronger in Penalty than in Prize. Similar predictions apply to inclusion errors, whereas the effect of the framing is ambiguous in the case of Exclusion errors (Propositions 7 and 8). Finally, as shown in Propositions 10 and 11, in the presence of loss aversion exclusion errors have a stronger disincentive effect on bids than inclusion errors in prize but not in penalty. ${ }^{18}$ As a consequence, the difference between the disincentive effects of exclusion and inclusion errors is larger in Prize than in Penalty.

Summing up, we test the following hypotheses about the effects of framing on bidding behavior in the presence of judgement errors:

H2a: In the absence of errors, bids are higher in Penalty than Prize.

H2b: The disincentive effects of adjudication errors are stronger in Penalty than Prize.

H2c: The disincentive effects of both exclusion and inclusion errors are stronger in Penalty than Prize.

H2d: The difference between the disincentive effects of exclusion and inclusion errors is larger in Prize than in Penalty.

\subsection{Participants and Procedures}

We recruited 256 participants among students at the University of Milan Bicocca using the ORSEE software (Greiner, 2004). Error-type treatments (Inclusion and Exclusion) were implemented within subjects. Framing treatments (Prize or Penalty) were implemented between subjects. We ran 8 sessions for each framing (Prize or Penalty) with 16 subjects per session. In each session, in either of the two framings, every subject faced all four error-type treatments (Exclusion x Inclusion), playing four phases of 10 rounds for each treatment (40 periods in total). In order to minimize the consequences of order effects, we implemented a cross-over design by reversing the order of the four treatments in each session as illustrated in Table 3 (two sessions were run for each of the four sequences of treatments).

In order to avoid repeated game effects, subjects were informed that groups would be randomly formed in each round. Unknown to subjects, groups of two subjects were formed in each round by randomly drawing from fixed sets of four subjects. We thus obtained four independent matching-group observations per session, for a total of 68 independent group-level observations overall.

\footnotetext{
${ }^{18}$ Reflection would also result in different predictions for the two framings. In particular, it can be shown that decreasing marginal utility operates in two opposite directions in the two framings so that exclusion (inclusion) has a stronger disincentive effect in Prize (Penalty).
} 
Table 3: Sequence of treatments, by session

\begin{tabular}{lcccc}
\hline & Phase 1 & Phase 2 & Phase 3 & Phase 4 \\
\hline Sequence 1 & T1 & T2 & T3 & T4 \\
Sequence 2 & T2 & T1 & T4 & T3 \\
Sequence 3 & T3 & T4 & T1 & T2 \\
Sequence 4 & T4 & T3 & T2 & T1 \\
\hline
\end{tabular}

In each session, upon arrival at the laboratory subjects were randomly assigned to computer terminals, located in cubicles that did not allow communication or visual interaction. To ensure public knowledge, instructions were distributed and read aloud separately for every treatment (see Appendix B for the instructions, translated from Italian). In order to check the understanding of the instructions subjects were asked to answer a set of control questions. The session started only after all subjects had answered the questions correctly. At the end of each round subjects were informed about both own and co-player's payoffs in that round. At the end of the experiment subjects were paid in cash according to their performance in one of the 40 periods, randomly selected.

The experiment was programmed and conducted with the software z-Tree (Fischbacher, 2007). Average earnings in the experiment were about 14 euros (including a participation fee of 2 euros), for sessions taking on average about 75 minutes.

\section{Results}

This section presents the experimental results. We start by providing an overview of bidding behavior across treatments. Next, we report test results for the effects of mis-judgement errors and the framing of the incentive scheme, respectively.

\subsection{Overview}

Figure 1 compares actual and theoretical bids, by treatment, pooling all subjects across the two framings. Observed bids are generally lower than theoretical bids, possibly reflecting the effects of repetition (see below). In the absence of judgement errors (T1), actual and theoretical bids are 445 and 500, respectively. Underbidding is also observed in the presence of exclusion error (T2, 308 vs. 375) and inclusion error (T3, 302 vs. 375). Actual and theoretical bids are instead relatively similar in the presence of adjudication errors (T4, 233 vs. 250). At a descriptive level, treatment effects are qualitatively consistent with the theoretical predictions. The presence of exclusion and inclusion errors, both jointly and individually, produces a substantial reduction in average bids. Average bids are relatively similar in the presence of exclusion and inclusion errors, respectively. The joint disincentive effect of the two types of errors is smaller than the sum of the individual effects of exclusion and inclusion errors.

Figure 2 illustrates the effects of repetition on bidding behavior within treatments, by comparing observed and predicted bids by treatment over 10 rounds 
Figure 1: Actual versus predicted bids, by treatment

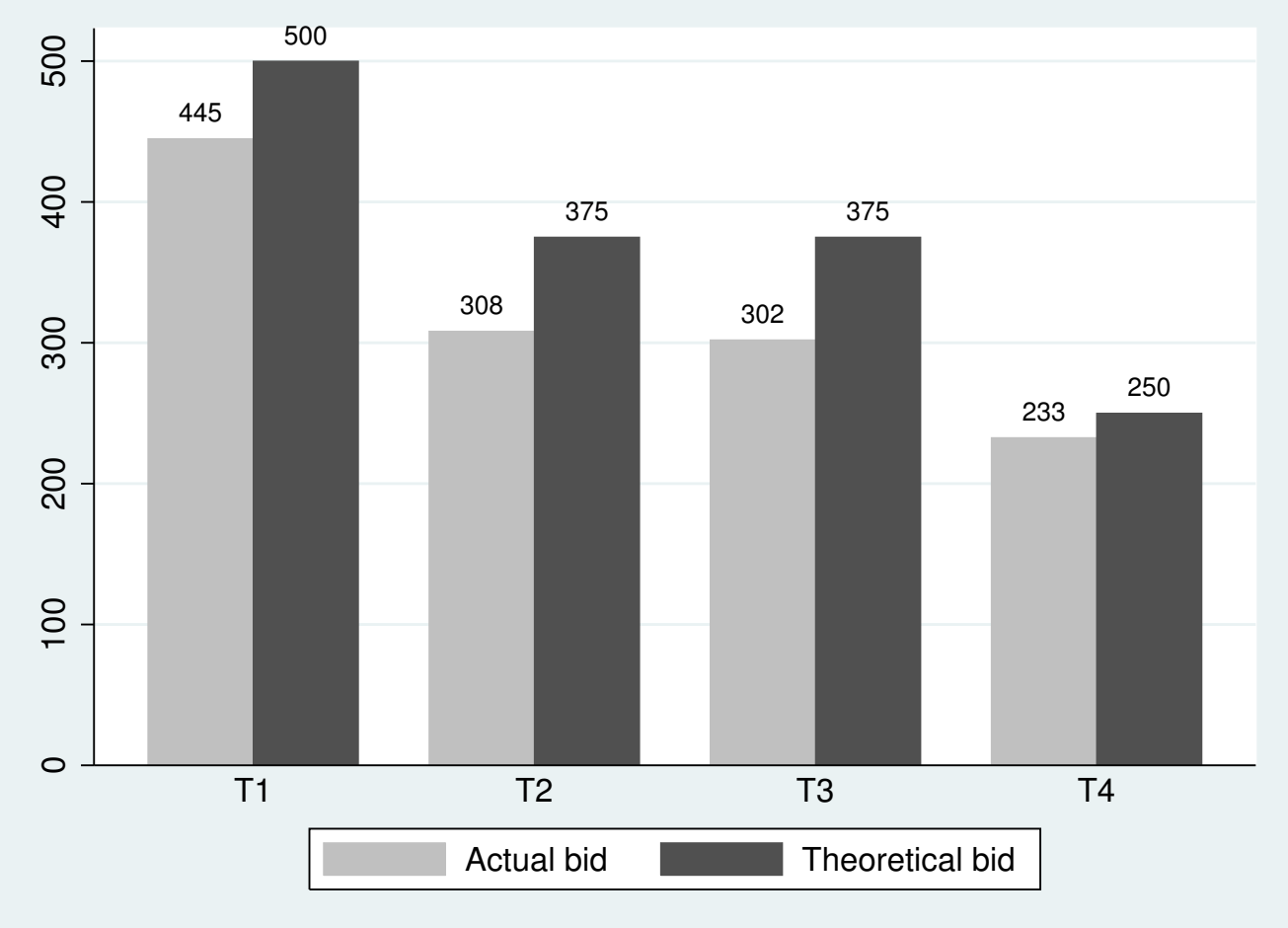

averaging across different phases (treatment-specific experience). Observed average bids follow a similar pattern in the four treatments: they are close to predicted bids in the initial rounds and decline over successive repetitions. The declining pattern within phases is more pronounced in $\mathrm{T} 2$ and $\mathrm{T} 3$, while relatively less strong in $\mathrm{T} 1$ and T4.

Figure 3 examines the effects of repetition on bidding behavior across phases, by comparing observed and predicted bids by treatment over 40 periods (overall experience). Average bids display a downward trend over rounds in T2 (exclusion) and T3 (inclusion), while the declining trend is absent in the absence of errors (T1) and less evident in the presence of adjudication errors (T4). Overall, these patterns indicate that the effects of repetition should be taken into account as they might interact with treatment effects.

\subsection{Effects of Mis-judgements on Effort}

In order to test the effects of mis-judgement errors on effort, we use a regression model that explains observed average group-level bids as a function of dummy variables for treatment effects (exclusion and inclusion errors) and their interaction:

$$
\bar{b}_{i, t}=\delta_{0}+\delta_{1} E X C_{i, t}+\delta_{2} I N C_{i, t}+\delta_{3} E X C * I N C_{i, t}+\beta \mathrm{x}_{i, t}+\epsilon_{i, t}
$$

where $\bar{b}_{i, t}$ denotes average bids (in Euro cents) in group $i$ and period $t$, with $i=1, \ldots, 64$ indicating independent matching groups and $t=1, \ldots, 40$ indicating periods. $E X C_{i, t}\left(I N C_{i, t}\right)$ is a dummy indicating whether group $i$ can face an exclusion 
Figure 2: Actual versus predicted bids over rounds, by treatment
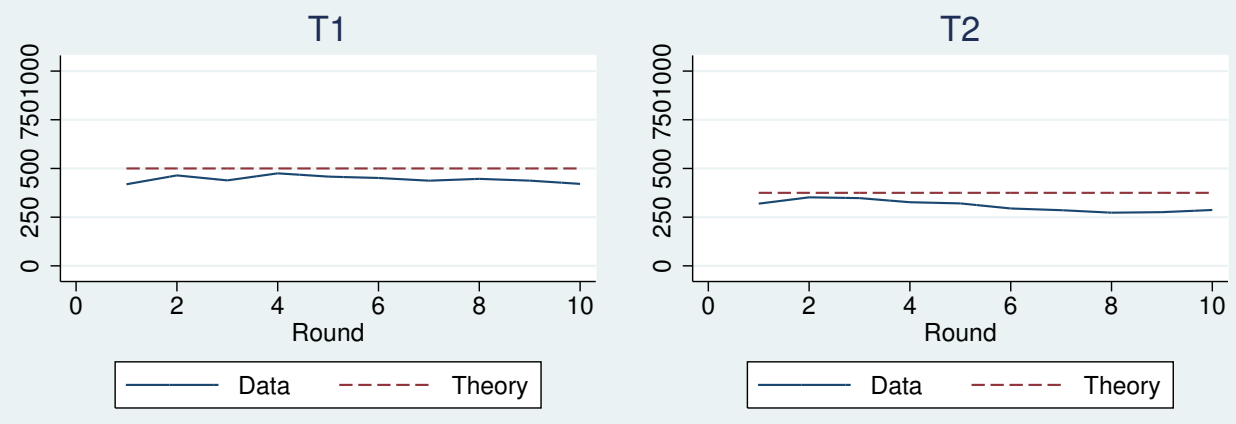

T3

$\mathrm{T} 4$
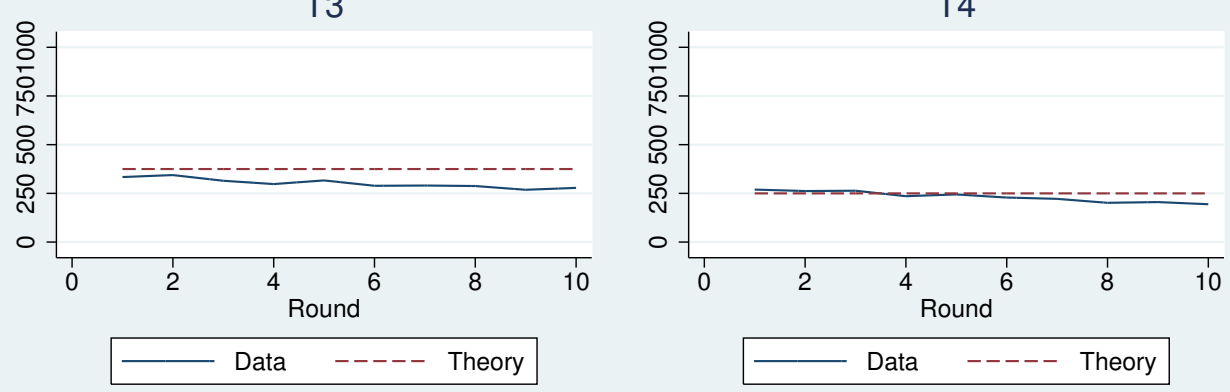

Figure 3: Actual versus predicted bids over periods, by treatment
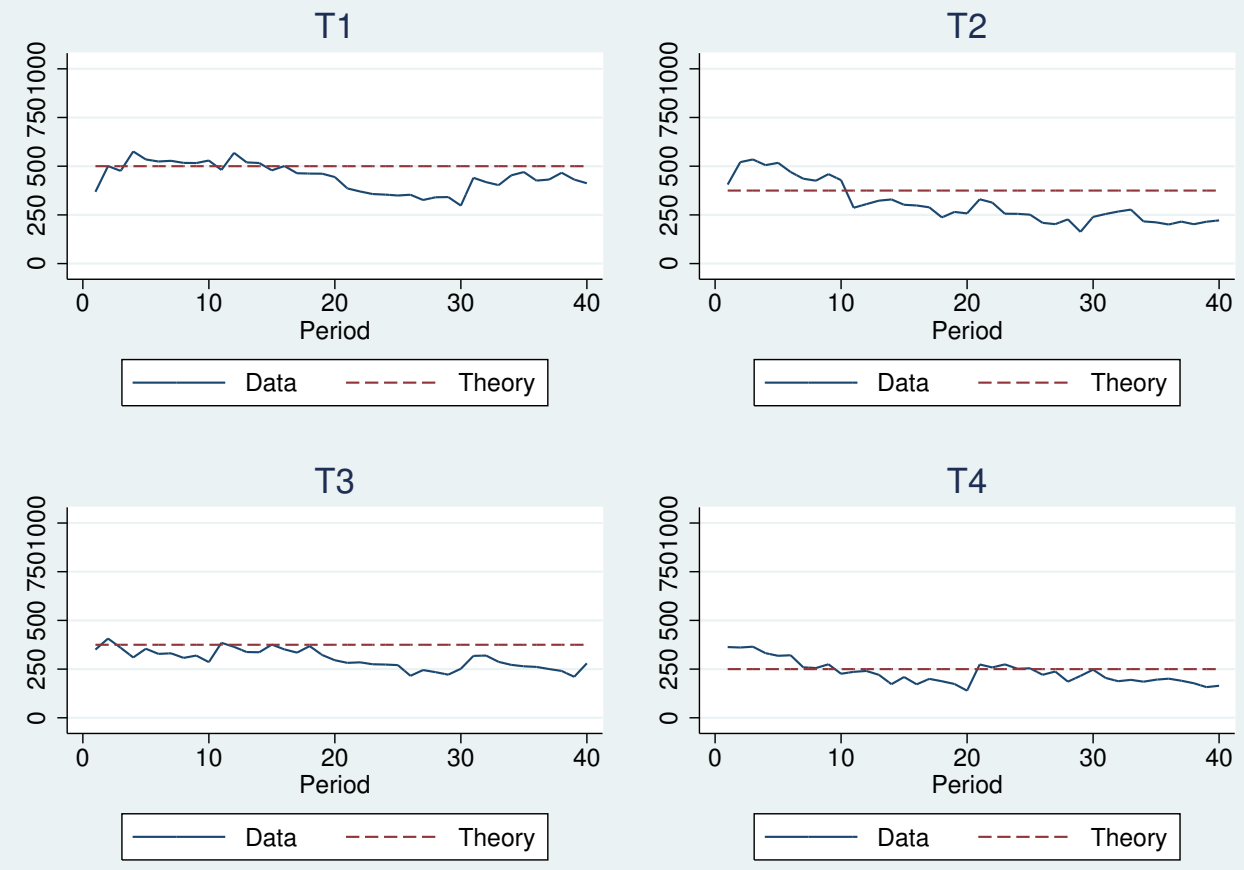

(inclusion) error in period t. $E X C_{i, t}$ is 1 in treatments $\mathrm{T} 2$ and $\mathrm{T} 4$ and 0 otherwise, while $I N C_{i, t}$ is 1 in treatments $\mathrm{T} 3$ and $\mathrm{T} 4$ and 0 otherwise. The vector $\mathrm{x}$ includes 
treatment-specific experience (a dummy that takes value 0 for the first five rounds within a treatment and 1 for the last five rounds), overall experience (a dummy that takes value 0 for the first 20 periods within a session and 1 for the last 20 periods) and a full set of session dummies. Standard errors are clustered by independent groups of four individuals.

Table 4 reports OLS estimates for alternative specifications of equation (1). Column (1) focuses on the joint effect of exclusion and inclusion errors, i.e. adjudication error $\left(\delta_{1}+\delta_{2}+\delta_{3}\right)$. The estimated effect of adjudication errors is large $(-212.3)$ and strongly significant. Column (2) reports the estimated effects of exclusion and inclusion errors, respectively, under the assumption of additivity $\left(\delta_{3}=0\right)$. Both exclusion and inclusion errors have a negative and significant effect on bids $\left(\hat{\delta}_{1}=-103.0\right.$ and $\hat{\delta}_{2}=-109.2$, respectively). Column (3) relaxes the assumption of additivity, indicating the presence of a negative interaction between exclusion and inclusion errors ( $\hat{\delta}_{3}=67.3$ has the opposite sign of the main effects). Therefore, exclusion and inclusion errors are substitutes, as the sum of the effects of the two error components is larger (in absolute value) than the effect of adjudication errors. Column (4) reports the estimate for the difference between the effects of exclusion and inclusion errors $\left(\delta_{1}-\delta_{2}\right)$. The difference is small (6.2) and not statistically significant. The null hypothesis that exclusion and inclusion errors have the same disincentive effect on bids cannot be rejected.

Table 4: Effects of judgement errors on effort

\begin{tabular}{|c|c|c|c|c|}
\hline & 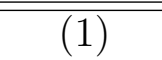 & $\overline{(2)}$ & 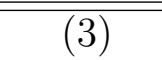 & (4) \\
\hline Adjudication & $\begin{array}{c}-212.3^{* *} \\
(18.3)\end{array}$ & & & \\
\hline Exclusion & & $\begin{array}{c}-103.0^{* *} \\
(13.8)\end{array}$ & $\begin{array}{c}-136.7^{* *} \\
(19.8)\end{array}$ & \\
\hline Inclusion & & $\begin{array}{c}-109.2^{* *} \\
(14.2)\end{array}$ & $\begin{array}{c}-142.9^{* *} \\
(21.0)\end{array}$ & \\
\hline Exclusion $*$ Inclusion & & & $\begin{array}{l}67.3^{*} \\
(28.2)\end{array}$ & \\
\hline Exclusion - Inclusion & & & & $\begin{array}{c}6.2 \\
(21.2)\end{array}$ \\
\hline $\mathrm{R} 2$ & 0.31 & 0.31 & 0.31 & 0.31 \\
\hline Number of observations & 2560 & 2560 & 2560 & 2560 \\
\hline
\end{tabular}

Note: dependent variable: average group-level bids in euro cents; OLS estimates; the set of regressors also includes dummy variables for treatment-specific experience and overall experience, and a full set of session dummy variables; standard errors (in parenthesis) clustered by independent matching group; * indicates $\mathrm{p}<0.05, * *$ indicates $\mathrm{p}<0.01$.

Table 5 assesses how repetition interacts with the effects of judgement errors, by comparing treatment effects between rounds 1-5 and 6-10 (treatment-specific experience), and between periods 1-20 and 21-40 (overall experience). Both overall and treatment-specific experience increase the size of the effect of adjudication errors. A similar result applies to the effects of both exclusion and inclusion errors. The 
interaction between exclusion and inclusion errors becomes stronger and statistically significant when subjects are more experienced. This indicates that the evidence of non-separability is reinforced by experience. Finally, the difference between the effects of exclusion and exclusion errors is positively related to overall experience (-74.2 and +52.3 in periods $1-20$ and $21-40$, respectively).

Table 5: Mis-judgements and effort, effects of repetition

\begin{tabular}{lcccc}
\hline \hline & Rounds 1-5 & Rounds 6-10 & Periods 1-20 & Periods 21-40 \\
\hline Adjudication & $-196.1^{* *}$ & $-228.4^{* *}$ & -134.1 & $-256.1^{* *}$ \\
& $(21.7)$ & $(19.7)$ & $(97.3)$ & $(70.9)$ \\
& & & & \\
Exclusion & $-117.8^{* *}$ & $-155.5^{* *}$ & $-118.5^{* *}$ & $-154.9^{* *}$ \\
& $(21.5)$ & $(23.4)$ & $(33.7)$ & $(20.3)$ \\
Inclusion & $-129.7^{* *}$ & $-156.0^{* *}$ & -44.3 & $-207.2^{* *}$ \\
& $(25.2)$ & $(23.1)$ & $(97.8)$ & $(60.0)$ \\
Exclusion * Inclusion & 51.5 & $83.1^{*}$ & 28.6 & $106.0^{* *}$ \\
& $(31.8)$ & $(31.4)$ & $(42.7)$ & $(35.5)$ \\
Exclusion - Inclusion & & & & \\
& $(22.8)$ & $(24.1)$ & $(105.2)$ & $(57.9)$ \\
\hline R2 & 0.31 & 0.32 & 0.31 & 0.28 \\
Observations & 1280 & 1280 & 1280 & 1280
\end{tabular}

Note: dependent variable: average group-level bids in euro cents; OLS estimates; the set of regressors also includes dummy variables for treatment-specific experience and overall experience, and a full set of session dummy variables; standard errors (in parenthesis) clustered by independent matching group; ${ }^{*}$ indicates $\mathrm{p}<0.05,{ }^{* *}$ indicates $\mathrm{p}<0.01$.

\subsection{Mis-judgements and the Framing of Incentives}

Figure 4 compares average bids in Prize and Penalty framings, respectively. Average bids are higher in the Penalty scheme in every treatment. As shown in Table 6, the difference in average bids between Penalty and Prize framings is statistically significant in the absence of errors (T1) and in the presence of adjudication errors (T4).

Table 7 compares the effects of judgement errors across framings. The effect of adjudication errors is relatively similar in Penalty and Prize framings (-206.5 and -218.1 euro cents, respectively). Consistently with the theoretical predictions, the disincentive effects of both exclusion and inclusion errors are stronger in Penalty than in Prize framings, although the difference is not statistically significant. The effects of exclusion and inclusion errors are small and not statistically significant in both Penalty and Prize framings.

To sum up, the main results about the effects of the framing of the incentive scheme are as follows: 
Figure 4: Average bids by framing, by treatment

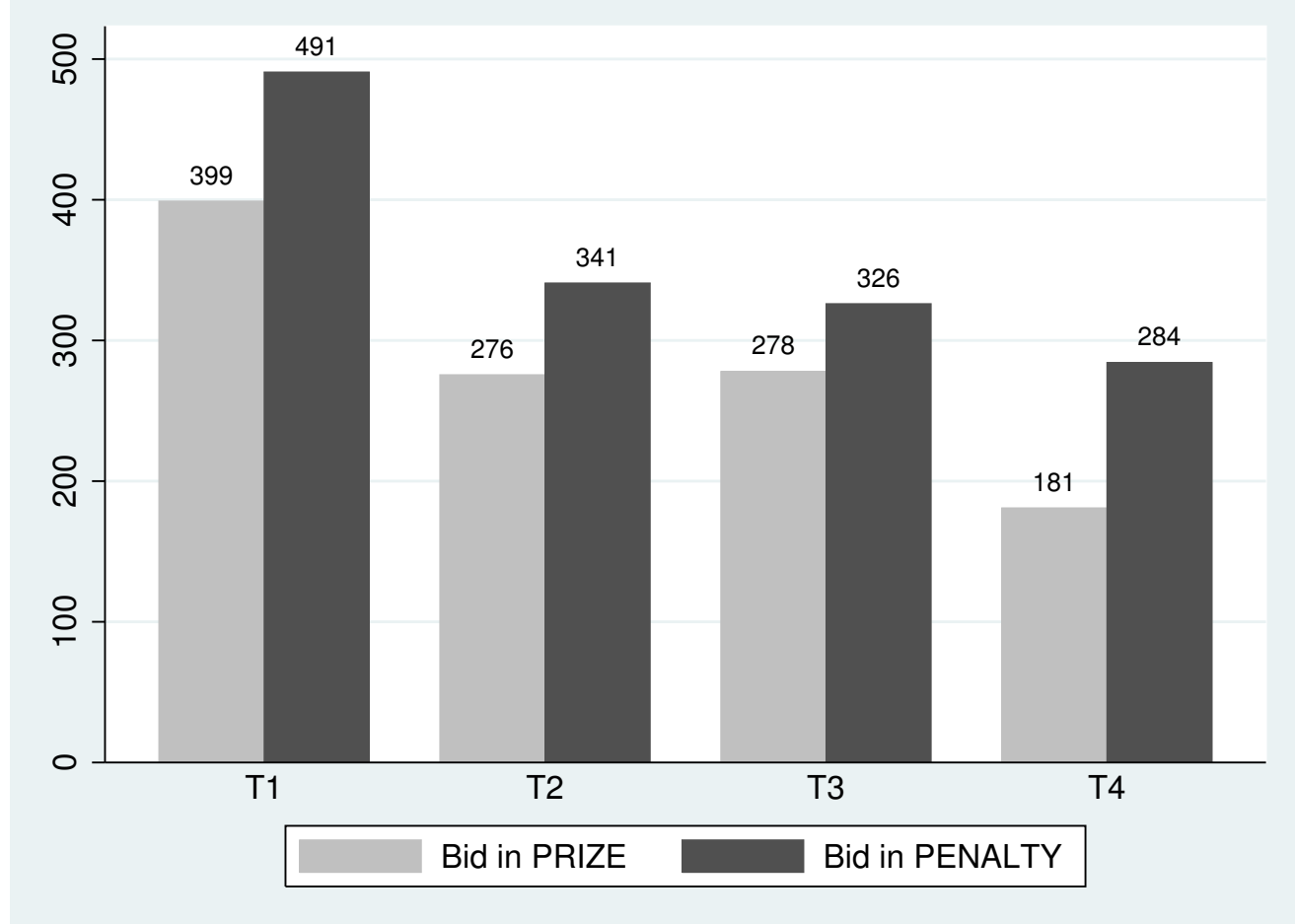

Table 6: Average bids by treatment and framing

\begin{tabular}{lccc}
\hline \hline & Penalty framing & Prize framing & Penalty-Prize \\
\hline T1 & $490.8^{* *}$ & $399.1^{* *}$ & $91.7^{*}$ \\
& $(31.6)$ & $(30.4)$ & $(43.5)$ \\
T2 & $340.9^{* *}$ & $275.6^{* *}$ & 65.2 \\
& $(33.3)$ & $(29.2)$ & $(44.0)$ \\
T3 & $326.1^{* *}$ & $278.0^{* *}$ & 48.2 \\
& $(30.4)$ & $(28.7)$ & $(41.4)$ \\
T4 & $284.3^{* *}$ & $181.0^{* *}$ & $103.4^{* *}$ \\
& $(33.0)$ & $(18.9)$ & $(37.7)$ \\
\hline$R^{2}$ & 0.76 & 0.72 & 0.74 \\
Number of observations & 1280 & 1280 & 2560
\end{tabular}

Note: dependent variable: average group-level bids in euro cents; OLS estimates; the set of regressors also includes dummy variables for treatment-specific experience and overall experience, and a full set of session dummy variables; standard errors (in parenthesis) clustered by independent matching group; $*$ indicates $\mathrm{p}<0.05,{ }^{* *}$ indicates $\mathrm{p}<0.01$.

1. In the absence of judgement errors, bids are higher in a Penalty than in a Prize incentive scheme.

2. In the presence of adjudication errors, bids are higher in a Penalty than in a Prize incentive scheme.

3. There is no significant interaction between the framing of the incentive mech- 
Table 7: Effects of mis-judgements, by framing

\begin{tabular}{lccc}
\hline \hline & Penalty framing & Prize framing & Penalty-Prize \\
\hline Adjudication & $-206.5^{* *}$ & $-218.1^{* *}$ & 11.6 \\
& $(25.2)$ & $(32.3)$ & $(40.6)$ \\
Exclusion & & & \\
& $-149.9^{* *}$ & $-123.4^{* *}$ & -26.5 \\
Inclusion & $(29.6)$ & $(26.5)$ & $(39.4)$ \\
& $-164.7^{* *}$ & $-121.1^{* *}$ & -43.6 \\
Exclusion * Inclusion & $(32.6)$ & $(35.3)$ & $(47.7)$ \\
& $108.1^{* *}$ & 26.4 & 81.7 \\
Exclusion - Inclusion & $(37.6)$ & $(41.4)$ & $(55.5)$ \\
& & & \\
\hline$R^{2}$ & 14.7 & -2.3 & 17.0 \\
Number of observations & $(39.7)$ & $(31.8)$ & $(50.5)$ \\
\hline
\end{tabular}

Note: dependent variable: average group-level bids in euro cents; OLS estimates; the set of regressors also includes dummy variables for treatment-specific experience and overall experience, and a full set of session dummy variables; standard errors (in parenthesis) clustered by independent matching group; ${ }^{*}$ indicates $\mathrm{p}<0.05,{ }^{* *}$ indicates $\mathrm{p}<0.01$.

anism and the effects of mis-judgements.

\section{Discussion and Conclusion}

We studied the disincentive effects of judgement errors in contests, focusing on the respective effects of the exclusion and inclusion components of adjudication errors. Theoretically, we have shown how behavioral aspects, such as risk aversion, loss aversion and the framing of the incentive scheme (prize vs. penalty) shape the effects of judgement errors on effort. Due to risk aversion, the negative effect of exclusion dominates the one of inclusion in both positive and negative incentive schemes. Loss aversion interacts with the effects of errors in different ways in the two reward schemes: in prize schemes exclusion dominates inclusion, while in penalty schemes exclusion has either the same or a less strong effect than inclusion. The comparison of bidding behavior across the two framings suggests that loss averse bidders bid more aggressively and perceive adjudication errors as more detrimental in a penalty scheme than in a prize scheme.

We tested experimentally the effects of an adjudication error, factorized in its exclusion and inclusion components, in the strategic context of an all-pay auction. The results indicate that adjudication errors negatively affect average bids in both positive and negative incentive schemes. Moreover, average bidding behavior is more aggressive in a penalty than in a prize scheme. However, in both reward schemes, the effects of exclusion and inclusion are not significantly different, in contrast with the implications of behavioral aspects like risk and loss aversion. Therefore, it is not 
the case that penalizing (or not rewarding) the best candidate (exclusion error) is perceived as more frustrating than not penalizing (or rewarding) the worst candidate (inclusion error). This insight can contribute to the design of (second best) optimal contests. Given that the two errors do not have a different disincentive effect on effort, it is more compelling to avoid the inclusion error as it is more costly for the contest organizer.

An additional finding of our experiment is that subjects bid more aggressively under penalty incentive schemes than under prize incentive scheme, something that has received relatively little attention in experimental literature on contests. This is an important result for the design of optimal incentives in competitive environments. ${ }^{19}$ Several recent studies have provided evidence that loss contracts - whereby penalties are assigned for not reaching a productivity target - increase workplace individual performance, both in the lab and in the field (Brooks, et al., 2011; Hossain and List, 2012; Fryer et al., 2012; Armantier and Boly, 2015). ${ }^{20}$ To the best of our knowledge, studies on the effects of gain and loss schemes on effort in competitive settings have limited their attention to work team contests. While in Dickinson (2001) prizes and fines are assigned to high and low contributors to the team goal, in Hong et al. (2015) rewards and punishments are assigned to the team overall, depending on its performance, and equally redistributed among its members. However, in both cases, individual effort can be driven by social concerns for the partners' payoffs that are completely absent in a context like the one we implemented, whereby competition occurs at the individual level.

Another aspect of our experimental findings that deserves consideration is that observed average bids overall are relatively close to risk neutral theoretical predictions. Instead, overbidding is a widespread phenomenon observed in many experiments on contests. ${ }^{21}$ However, it is worth noticing that overbidding mostly characterizes experimental lottery contests, while for experimental all-pay auctions, the evidence is mixed. ${ }^{22}$ Most importantly, as discussed by Sheremeta (2013), overbidding rates may depend on a series of characteristics of the experimental design. Factors like the number of bidders, the payoff structure (prize and endowment relative sizes), the bidders' experience (number of repetitions) and the matching protocol

\footnotetext{
${ }^{19}$ We are aware of only a recent experiment by Chowdhury et al. (2016) on the effects of property rights on contest outcomes under loss aversion, that implement a similar manipulation on the reward structure. Specifically, in their gain treatment two players start with no prize and bid and whoever wins, gets a prize. In their loss treatment two players start with own prizes and bid and whoever loses, loses his prize. Even if theoretically there should be no difference in bids in these two treatments, the authors find that subjects bid more in the loss treatment.

${ }^{20}$ In contrast, in a different context Levitt et al. (2012) find that the framing of educational incentives as gains or losses does not significantly affect effort.

${ }^{21}$ See for example Millner and Pratt (1989), Davis and Reilly (1988), Potters et al. (1998), Sheremeta and Zhang (2010), Corazzini et al. (2010), Faravelli and Stanca (2012), Price and Sheremeta (2015) among many others.

${ }^{22}$ For example, Potters et al. (1998) compares behavior in a lottery contest and in an all pay auction and finds over-dissipation only in the former.
} 
(anonymous or not, repeated or random) can influence the overbidding rate. ${ }^{23}$

Among the existing experimental studies on symmetric auctions, the closest to ours in terms of payoff structure is Gneezy and Smorodinsky (2006). ${ }^{24}$ Yet, while in our experiment bidders receive an endowment in every round, in their experiment bidders receive a large endowment one time at the beginning of the experiment. ${ }^{25}$ Thus, our subjects may perceive a more salient cap on their bids in each round and this may be the reason why we observe lower average bids. Moreover, in their experiment bidders are at least four. ${ }^{26}$ As the number of bidders is widely recognized to have a positive effect on overbidding, ${ }^{27}$ this additional difference in the design may contribute to explain the differences with our findings.

The existence of a cap on bids is one of the arguments provided by Potters et al. (1998) to explain why they do not find strong evidence in favor of overbidding in an all-pay auction setting that shares some crucial characteristics with ours: the same number of bidders, the same matching protocol (random and anonymous matching), and a large number of rounds that is at least as twice as in other studies (30 in their experiment). In every round subjects are endowed with an amount of money that is slightly above the prize value and this cap may exert a stronger pressure on average bids with respect to Gneezy and Smorodinsky (2006) design, making Potters et al. (1998) results more similar to ours.

Our paper sheds light on the underlying mechanisms through which adjudication errors in contests affect effort and it opens up fruitful avenues for future research, as errors can affect many relevant strategic contexts (e.g., labor market, education, scientific dissemination, political lobbying or elections, etc.) and have a significant economic impact. Moreover, in many competitions (e.g., for bonuses in the workplace or job positions) contested resources are not always available in fixed amounts. There might be some uncertainty at the competition stage on whether such resources will either remain the same, abound or vanish at the awarding stage, especially in periods of high economic volatility. Our study opens up the way for analyzing contests that incorporate uncertainty over the amount of available resources.

\footnotetext{
${ }^{23} \mathrm{~A}$ subset of papers within this literature specifically investigate the overbidding phenomenon in contests manipulating some of these design characteristics and find for example that: the larger the number of bidders, the higher the overbidding rate (probably due to a lower collusion among bidders); the lower the gap between the endowment and the prize value, the stronger the downward pressure on bidding, thus the lower is the overbidding rate.

${ }^{24}$ Other experimental studies on symmetric all pay auctions are Ong and Chen (1998), Ernst and Thoni (2013), Lugovsky et al. (2010), Klose and Sheremeta (2012).

${ }^{25}$ In Gneezy and Smorodinsky (2006) design, subjects have an endowment of 1000 ECU spread over 10 rounds and the prize in each round is $100 \mathrm{ECU}$.

${ }^{26}$ Like in our experiment Gneezy and Smorodinsky (2006) adopt a random anonymous matching.

${ }^{27}$ In fact, Gneezy and Smorodinsky (2006) study the effect of the number of bidders on overbidding rates, implementing an all-pay auctions with four, eight and twelve bidders.
} 


\section{References}

Armantier, O., Boly, A. 2015. Framing of incentives and effort provision. International Economic Review, 56(3), 917-938.

Baye, M.R., Kovenock, D., de Vries, C.G., 1996. The all-pay auction with complete information. Economic Theory, 8(2), 291-306.

Brooks, R. R. W., Stremitzer, A. and Tontrup, S., 2012. Framing contracts: Why loss framing increases effort. Journal of Institutional and Theoretical Economics, 168(1), 62-82.

Chowdhury, S.M., Jeon, J.Y., Ramalingam, A., 2016. Property rights and loss aversion in contests. mimeo.

Corazzini, L., Faravelli, M., Stanca, L. 2010. A prize to give for: An experiment on public good funding mechanisms, The Economic Journal, 120, 944967.

Davis, D., Reilly, R.J., 1998. Do too many cooks always spoil the stew? An experimental analysis of rent-seeking and the role of a strategic buyer. Public Choice, pp. 89115.

Dickinson, D.L., 2001. The carrot vs the stick in work team motivation. Journal of Experimental economics 4(1), 107-124.

Ernst, C., Thöni, C., 2013. Bimodal bidding in experimental all-pay auctions. Games, 4(4), 608-623.

Eckel, C., Grossman, P., 2002. Sex Differences and Statistical Stereotyping in Attitudes toward Financial Risk. Evolution and Human behavior 23(4), pp. 281-295.

Faravelli, M., Stanca, L. (2012), Single versus multiple prize contests to finance public goods: Theory and experimental evidence. Journal of Economic Behavior and Organization, 81, 677-688.

Fischbacher, U., 2007. z-Tree: Zurich toolbox for ready-made economic experiments Experimental Economics, 10(2), 171-178.

Fryer Jr, R. G., Levitt, S. D., List, J. A., and S. Sadoff. 2012. Enhancing the efficacy of teacher incentives through loss aversion: A field experiment. NBER Working Paper No. 18237.

Gächter, S., Johnson, E.J., Herrmann, A., 2007. Individual-level loss aversion in riskless and risky choices. CeDEx Discussion Paper No. 2007.

Gneezy, U., Smorodinsky, R., 2006. All-pay auctions - An experimental study. Journal of Economic Behavior and Organization, 61, 255-275.

Greiner, B. 2004. The online recruitment system ORSEE 2.0 - A guide for the organization of experiments in economics. Department of Economics, University of Cologne mimeo. 
Hammond, K.R., 1996. Human judgment and social policy. Irreducible uncertainty, inevitable error, unavoidable injustice. Oxford University Press, New York.

Hillman, A., Riley, J.G., 1989. Politically contestable rents and transfers. Economics and Politics, 1, 17-40.

Hong, F., Hossain, T. and List, J. A., 2015. Framing manipulations in contests: A natural field experiment. Journal of Economic Behavior and Organization, 128, 372-382.

Hossain, T. and List, J. A., 2012. The behavioralists visits the factory: Increasing productivity using simple framing manipulations. Management Science, 58(12), 2151-2167.

Kahneman, D., Tversky, A., 1979. Prospect Theory: An analysis of decision under risk. Econometrica 47(2), 263-292.

Kaplow, L., Shavell, S., 1994. Accuracy in the determination of liability. Journal of Law and Economics, 37, 1-15.

Klose, B., Sheremeta, R.M., 2012. Behavior in all-pay and winner-pay auctions with identity-dependent externalities. Working Paper.

Konrad, K., 2009. Strategy and dynamics in contests, Oxford University Press, New York.

Lazear, E.P., Rosen, S., 1981. Rank and order tournament: An optimal labor contract. Journal of Political Economy, 89(5), 841-864.

Levitt, S.D., List, J.A., Neckermann, S., Sadoff, S., 2012. The behavioralist goes to school: Leveraging behavioral economics to improve educational performance. NBER Working Paper No. 18165.

Lugovskyy, V., Puzzello, D., Tucker, S., 2010. An experimental investigation of overdissipation in the all pay auction. European Economic Review, 54(8), 974997.

Millner, E.L., Pratt, M.D., 1989. An experimental investigation of efficient rentseeking. Public Choice, 62(2), 139-151.

Ong, D., Chen, Z., 2012. Tiger women: An all-Pay auction experiment on the gender heuristic of the desire to win. Working Paper.

Png, Ivan P. L., 1986. Optimal subsidies and damages in the presence of judicial error. International Review of Law and Economics, 6, 101-105.

Polinsky, A. M., Shavell, S., 2009. Public enforcement of law, volume 3. Amsterdam: Edward Elgar.

Potters, J., de Vries, C., van Winden, F., 1998. An experimental investigation of rational rent seeking. European Journal of Political Economy, 14(4), 783-800. 
Price, C., Sheremeta, R. Endowment origin, demographic effects and individual preferences in contests. Journal of Economics and Management Strategy, 24, 597619.

Rizzolli, M., Stanca, L., 2012. Judicial errors and crime deterrence: Theory and experimental evidence. Journal of Law and Economics, 55(2), 311-338.

Sheremeta, R., 2013. Overbidding and heterogeneous behavior in contest experiments. Journal of Economic Surveys, 27, 491-514.

Sheremeta, R., Zhang, J., 2010. Can groups solve the problem of over-bidding in contests? Social Choice and Welfare, 35, 175-197.

Thorngate, W., Carroll, B., 1990. Tests versus contests. Recent Research in Psychology, 431-438.

Thorngate, W., Dawes, R.M., Foddy, M., 2009. Judging merit. Psychology Press, New York.

Tullock, G., 1980. Efficient rent-seeking. In Buchanan, J.M., Tollison, R.D. and Tullock, G. (Eds.), Toward a theory of the rent-seeking society, 97-112. College Station: Texas A\&M University Press. 


\section{Appendix A: Proofs}

It can be easily shown that in none of the all-pay auctions considered in Section 2 there exists a Nash equilibrium in pure strategies. Let us provide some notation that we will adopt in the following proofs. Let $\underline{b}_{i}$ and $\bar{b}_{i}$ denote the lower and upper bound of player i's equilibrium bid distribution $F_{i}(\cdot)$. Also, let $\alpha_{i}(\cdot)$ denote the size of a mass point in $i$ 's distribution. Let $U_{i}\left(b, F_{j}(\cdot)\right)$ be player $i$ 's expected utility from bid $b$ given the strategy of the opponent $j, F_{j}(\cdot)$.

In order to construct the mixed-strategy Nash equilibrium of each error setting, we first obtain the lower and the upper bounds of the supports of the two mixed strategies (points 1 and 2 below); next, we prove that equilibrium mixed strategies are continuous (point 3 ); finally, we derive the symmetric equilibrium cdf $F(\cdot)$.

Proof of Proposition 1. The proof goes along the following steps:

1. We prove that the supports of the two equilibrium mixed strategies have the same lower bound, which is equal to zero, i.e., $\underline{b}_{1}=\underline{b}_{2}=0$, and that there is no atom in zero, i.e., $F_{i}(0)=0, i=1,2$.

(a) We first show that the lower bounds must coincide, i.e., $\underline{b}_{1}=\underline{b}_{2}$. Bids lower than zero are ruled out a priori. Suppose that the lower bound of either player, say player 2 , is larger, i.e., $0 \leq \underline{b}_{1}<\underline{b}_{2}$. Player 1 will put no density on the interval $\left[\underline{b}_{1}, \underline{b}_{2}\right)$ because the probability of winning is zero. Thus, for player 2 is profitable to deviate to a lower bid $b<b_{2}$, whereby, as long as $b>b_{1}$, the probability of winning is the same as in $\underline{b}_{2}$, but the payoff is larger.

(b) Secondly, we show that lower bounds are equal to zero, i.e., $\underline{b}_{1}=\underline{b}_{2}=0$. Suppose that $\underline{b}_{1}=\underline{b}_{2}=\underline{b}>0$. We first argue that $\underline{b}_{1}=\underline{b}_{2}=\underline{b}$ implies that at least one player puts no mass on $\underline{b}$. Suppose that both $F_{1}(\underline{b})$ and $F_{2}(\underline{b})$ are larger than zero. Then, either player, say player 1 , could profitable deviate to $\underline{b}+\epsilon$ so to increase the probability of winning by a finite amount. Secondly, we argue that it cannot be the case that only one player, say player 1 , puts no mass on $\underline{b}$, i.e., $F_{1}(\underline{b})=0$. Indeed, if this is the case, then player 2 incurs in a certain loss at $\underline{b}$ and, thus, has the incentive to shift the mass away. Thus, it must be the case that both $F_{2}(\underline{b})$ and $F_{1}(\underline{b})$ are equal to zero. Finally, we argue that $F_{2}(\underline{b})=F_{1}(\underline{b})=0$ implies that $\underline{b}_{1}=\underline{b}_{2}=0$. If no player puts mass on the lower bound $\underline{b}$, then either player has the incentive to deviate to a strategy that puts mass on zero, where the probability of winning is the same as in $\underline{b}$ but the payoff is larger. Thus, it must be the case that $\underline{b}=0$.

(c) Third, we show that no player has an atom in zero, i.e., $\alpha_{i}(0)=0, i=1,2$. Suppose that $\alpha_{1}(0)>0$ and $\alpha_{2}(0)>0$. Either player, say player 2 , can relocate $\alpha_{2}(0)$ on $\epsilon>0$ so to increase the probability of winning by a finite amount. Thus, it cannot be the case that both players bid zero with strictly positive probability.

Suppose that only one player, say player 2 , bids zero with strictly positive probability, i.e., $\alpha_{1}(0)=0$ and $\alpha_{2}(0)>0$. Player 2 has an incentive to deviate and shift mass of size $\alpha_{2}(0)$ to $\epsilon>0$ so to increase the probability of winning by a finite amount. 
2. Next, we prove that the supports of the two equilibrium mixed strategies have the same upper bound, i.e., $\overline{b_{1}}=\overline{b_{2}}=\pi$.

(a) We first show that the upper bounds coincide, i.e., $\overline{b_{1}}=\overline{b_{2}}$. Suppose that, the upper bound of either player, say player 1 , is larger, i.e., $\overline{b_{2}}<\overline{b_{1}} \leq \pi$. Then, player 1 finds it profitable to deviate to a lower upper bound where the probability of winning is the same as in $\overline{b_{1}}$, but the payoff is larger. Hence, it must be the case that $\overline{b_{1}}=\overline{b_{2}}$.

(b) Secondly, we show that the upper bounds must be equal to $\pi$. Suppose that $\overline{b_{1}}=\overline{b_{2}}=\bar{b}<\pi$. Then, player 1 finds it profitable to bid $b_{1}$ slightly above $\overline{b_{1}}$, as long as $\pi-b_{1}$ is positive, so to win with certainty. Thus, it must be the case that $\overline{b_{1}}=\overline{b_{2}}=\pi$.

3. We now prove that the equilibrium is a continuous mixed strategy equilibrium, that is no bid in the support is played with strictly positive probability.

Suppose there exists a bid $b$ in the support of either player's mixed strategy, say player 1 's, such that $\alpha_{1}(b)>0$. This implies that $F_{1}(\cdot)$ is discontinuous at $b$. Hence, there is some $\epsilon>0$ arbitrarily small such that player 2 puts no density on the interval $[b-\epsilon, b)$. Thus, player 1 would be better off by bidding slightly less than $b$ obtaining the same probability of winning and a larger payoff.

Suppose that there exists a bid $b$ on which both players put strictly positive probability. Then, either of the two can profitably deviate by shifting mass from $b$ to $b+\epsilon$ so to increase the probability of winning by a finite amount.

4. Points 1 to 3 have shown that both players randomize continuously on the support $[0, \pi]$. Now, we compute the equilibrium mixed strategy profile. As any bid in the support should provide the same expected utility, we impose that for $i=1,2, j \neq i$ :

$$
U_{i}\left(b, F_{j}(\cdot)\right)=U_{i}\left(0, F_{j}(\cdot)\right) \Longleftrightarrow F_{j}(b) \pi-b_{i}=0
$$

Hence, for every $j=1,2, F_{j}(b)=\frac{b}{\pi}$. Notice that the probability of a tie is zero for any bid in the support.

Proof of Proposition 2. The proof coincides with the proof of Proposition 2 up except for point 2 and 4 . Thus, we elaborate points 2 and 4 only.

1. As point 1 in Proof of Proposition 1.

2. Next, we prove that the supports of the two equilibrium mixed strategies have the same upper bound, i.e., $\overline{b_{1}}=\overline{b_{2}}=(1-p) \pi$.

(a) We first show that the upper bounds coincide, i.e., $\overline{b_{1}}=\overline{b_{2}}$. Suppose that, the upper bound of either player, say player 1 , is larger, i.e., $\overline{b_{2}}<\overline{b_{1}} \leq$ $(1-p) \pi$. Then, player 1 finds it profitable to deviate to a lower upper bound where the probability of winning is the same as in $\overline{b_{1}}$ (i.e., $1-p$ ), but the payoff is larger. Hence, it must be the case that $\overline{b_{1}}=\overline{b_{2}}$. 
(b) Secondly, we show that the upper bounds must be equal to $(1-p) \pi$. Suppose that $\overline{b_{1}}=\overline{b_{2}}=\bar{b}<(1-p) \pi$. Then, either player finds it profitable to bid $b$ slightly above $\bar{b}$, as long as the expected return $(1-p) \pi-b$ is positive, so to increase the probability of winning by a finite amount.

Suppose that $\bar{b}_{1}=\bar{b}_{2}=\bar{b}>(1-p) \pi$. Then, either player by bidding $\bar{b}$ would get the prize with probability $(1-p)$ and encur in a loss. Hence, it must hold that $\overline{b_{1}}=\overline{b_{2}}=(1-p) \pi$.

3. As point 3 in Proof of Proposition 1.

4. Points 1 to 3 have shown that both players randomize continuously on the support $[0,(1-p) \pi]$. Now, we compute the equilibrium mixed strategy profile. As any bid in the support should provide the same expected utility, we impose that for $i=1,2, j \neq i$ :

$$
U_{i}\left(b, F_{j}(\cdot)\right)=U_{i}\left(0, F_{j}(\cdot)\right) \Longleftrightarrow(1-p) F_{j}(b) \pi-b=0
$$

Hence, for every $j=1,2, F_{j}(b)=\frac{b}{(1-p) \pi}$. Notice that the probability of a tie is zero for any bid in the support.

Proof of Proposition 3. To prove the result of Proposition 3 we follow the previous proofs. However, since some arguments are slightly different, we report them for completeness.

1. We prove that the supports of the two equilibrium mixed strategies have the same lower bound, which is equal to zero, i.e., $\underline{b}_{1}=\underline{b}_{2}=0$, and that there is no atom in zero, i.e., $F_{i}(0)=0, i=1,2$.

(a) We first show that the lower bounds must coincide, i.e., $\underline{b}_{1}=\underline{b}_{2}$. Bids lower than zero are ruled out a priori. Suppose that the lower bound of either player, say player 2 , is larger, i.e., $0 \leq \underline{b}_{1}<\underline{b}_{2}$. Player 1 will put no density on the interval $\left(\underline{b}_{1}, \underline{b}_{2}\right)$ because the expected utility from any bid $b_{1}$ in this interval is lower than the expected utility from $\underline{b}_{1}$, as $p \pi-b_{1}<p \pi-\underline{b}_{1}$. Thus, for player 2 is profitable to deviate to a lower bid $b_{2}<\underline{b}_{2}$, whereby, as long as $b_{2}>\underline{b}_{1}$, the probability of winning is the same as in $\underline{b}_{2}$ (i.e., $\alpha_{1}\left(\underline{b}_{1}\right)$ ), but the payoff is larger.

(b) Secondly, we show that lower bounds are equal to zero, i.e., $\underline{b}_{1}=\underline{b}_{2}=0$. Suppose that $\underline{b}_{1}=\underline{b}_{2}=\underline{b}>0$. We first argue that $\underline{b}_{1}=\underline{b}_{2}=\underline{b}$ implies that at least one player puts no mass on $\underline{b}$. Suppose that both $F_{1}(\underline{b})$ and $F_{2}(\underline{b})$ are larger than zero. Then, either player, say player 1 , could profitable deviate to $\underline{b}+\epsilon$ so to increase the probability of winning by a finite amount. Secondly, we argue that it cannot be the case that only one player, say player 1 , puts no mass on $\underline{b}$, i.e., $F_{1}(\underline{b})=0$. Indeed, if this is the case, then player 2 has the incentive to shift the mass from $\underline{b}$ to $\underline{b}+\epsilon$ so to increase the probability of winning by a finite amount. Thus, it must be the case that both $F_{2}(\underline{b})$ and $F_{1}(\underline{b})$ are equal to zero. Finally, we argue that $F_{2}(\underline{b})=F_{1}(\underline{b})=0$ implies that $\underline{b}_{1}=\underline{b}_{2}=0$. If no player puts mass on the lower bound $\underline{b}$, then either player has the incentive to 
deviate to a strategy that puts mass on zero, where the probability of winning is the same as in $\underline{b}$, i.e., $p$, but the payoff is larger. Thus, it must be the case that $\underline{b}=0$.

(c) Third, we show that no player has an atom in zero, i.e., $\alpha_{i}(0)=0, i=1,2$. Suppose that $\alpha_{1}(0)>0$ and $\alpha_{2}(0)>0$. Either player, say player 2, can relocate $\alpha_{2}(0)$ on $\epsilon>0$ so to increase the probability of winning by a finite amount. Thus, it cannot be the case that both players bid zero with strictly positive probability.

Suppose that only one player, say player 2 , bids zero with strictly positive probability, i.e., $\alpha_{1}(0)=0$ and $\alpha_{2}(0)>0$. Player 2 has an incentive to deviate and shift mass of size $\alpha_{2}(0)$ to $\epsilon>0$ so to increase the probability of winning by a finite amount.

2. Next, we prove that the supports of the two equilibrium mixed strategies have the same upper bound, i.e., $\overline{b_{1}}=\overline{b_{2}}=(1-p) \pi$, with an argument similar to the one of point 2 of Proposition 1.

(a) We first show that the upper bounds coincide, i.e., $\overline{b_{1}}=\overline{b_{2}}$. Suppose that, the upper bound of either player, say player 1 , is larger, i.e., $\overline{b_{2}}<$ $\overline{b_{1}} \leq(1-p) \pi$. Then, player 1 finds it profitable to deviate to a lower upper bound where the probability of winning is the same as in $\overline{b_{1}}$, but the payoff is larger. Hence, it must be the case that $\overline{b_{1}}=\overline{b_{2}}$.

(b) Secondly, we show that the upper bounds must be equal to $(1-p) \pi$.

Suppose that $\overline{b_{1}}=\overline{b_{2}}=\bar{b}<(1-p) \pi$. Then, either player finds it profitable to bid $b$ slightly above $\bar{b}$, as long as the expected return $(1-p) \pi-b$ is positive, so to win with certainty.

Suppose that $\bar{b}_{1}=\bar{b}_{2}=\bar{b}>(1-p) \pi$. Then, either player by bidding $\bar{b}$ would get the prize with certainty and obtain a payoff $(\pi-\bar{b})$ which is less than what he would get from bidding zero $(p \pi)$. Hence, it must hold that $\overline{b_{1}}=\overline{b_{2}}=(1-p) \pi$.

3. As point 3 in Proof of Proposition 1.

4. Points 1 to 3 have shown that both players randomize continuously on the support $[0,(1-p) \pi]$. Now, we compute the equilibrium mixed strategy profile. As any bid in the support should provide the same expected utility, we impose that for $i=1,2, j \neq i$ :

$$
U_{i}\left(b, F_{j}(\cdot)\right)=U_{i}\left(0, F_{j}(\cdot)\right) \Longleftrightarrow(1-p) F_{j}(b) \pi+p \pi-b=p \pi
$$

Hence, for every $j=1,2, F_{j}(b)=\frac{b}{(1-p) \pi}$. Notice that the probability of a tie is zero for any bid in the support.

Proof of Proposition 4. To prove this result, we can use the arguments contained in the previous proofs. In particular, to prove that lower bounds must be the same and are equal to zero, we can replicate points 1 and 3 of the proof of Proposition 
3. The expected utility in an adjudication error setting is indeed equal to (1 $2 p) F(b) \pi+p \pi-b$, while in an inclusion error setting is equal to $(1-p) F(b) \pi+p \pi-b$. Similarly, the same argument for upper bounds used in the proof of Proposition 3 applies here. However, now the upper bounds coincide with $(1-2 p) \pi$ as for any bid above this threshold bidders would get less than what they get from bidding zero (i.e., $p \pi)$. As any bid in the support $[0,(1-2 p) \pi]$ should provide the same expected utility, we impose that for $i=1,2, j \neq i$ :

$$
U_{i}\left(b, F_{j}(\cdot)\right)=U_{i}\left(0, F_{j}(\cdot)\right) \Longleftrightarrow(1-2 p) F_{j}(b) \pi+p \pi-b=p \pi
$$

Hence, for every $i, F_{i}(b)=\frac{b}{(1-2 p) \pi}$.

Proof of Proposition 5. Denote $\bar{b}^{E}$ and $\bar{b}^{I}$ the upper bounds of the support of $F^{E}(\cdot)$ and $F^{I}(\cdot)$ respectively. As $b$ takes only positive values, we derive the expected bids in the two settings from the survival function and obtain:

$$
E^{E}(b)=\int_{0}^{\bar{b}^{E}}\left[1-F^{E}(b)\right] d b \leq \int_{0}^{\bar{b}^{I}}\left[1-F^{I}(b)\right] d b=E^{I}(b) .
$$

It holds true that

$$
\begin{aligned}
F^{E}(b)= & \frac{u(w)-u(w-b)}{(1-p)[u(w+\pi-b)-u(w-b)]} \geq \\
& \frac{(1-p)[u(w)-u(w-b)]+p[u(w+\pi)-u(w+\pi-b)]}{(1-p)[u(w+\pi-b)-u(w-b)]}=F^{I}(b)
\end{aligned}
$$

since

$$
u(w)-u(w-b) \geq u(w+\pi)-u(w+\pi-b)
$$

due to concavity of $u(\cdot)$.

To show that $\bar{b}^{E}<\bar{b}^{I}$ we first consider that $\bar{b}^{E}$ solves:

$$
(1-p) u\left(w+\pi-\bar{b}^{E}\right)+p u\left(w-\bar{b}^{E}\right)=u(w)
$$

where the left hand side is the expected utility from bidding exactly $\bar{b}^{E}$ and thus winning, while the right hand side is the expected utility from bidding 0 and losing.

Similarly, $\bar{b}^{I}$ solves the indifference condition:

$$
u\left(w+\pi-\bar{b}^{I}\right)=p u(w+\pi)+(1-p) u(w) .
$$

It is enough to find some bid $b$ in the support of $F^{I}(\cdot)$ such that condition A.13 evaluated in $b$ becomes:

$$
(1-p) u(w+\pi-b)+p u(w-b)<u(w)
$$

Consider $b=(1-p) \pi$. Observe that $\bar{b}^{I}>(1-p) \pi$. Suppose not, that is, suppose that $\bar{b}^{I} \leq(1-p) \pi$. We can exclude that $\bar{b}^{I}=(1-p) \pi$-as it does not satisfy condition A.14. Then, bidding $(1-p) \pi$ should make bidders worse off than bidding 0 , that is 


$$
u(w+\pi-(1-p) \pi)<p u(w+\pi)+(1-p) u(w)
$$

so that

$$
u(w+p \pi)<p u(w+\pi)+(1-p) u(w) .
$$

By definition of risk aversion

$$
p u(w+\pi)+(1-p) u(w) \leq u(p(w+\pi)+(1-p) w)=u(w+p \pi)
$$

which leads to a contradiction. Thus, it must be the case that $\bar{b}^{I}>(1-p) \pi$. Secondly, we observe that $\bar{b}^{E} \leq(1-p) \pi$ which implies that:

$$
(1-p) u(w+\pi-(1-p) \pi)+p u(w-(1-p) \pi) \leq u(w) .
$$

Indeed, by definition of risk aversion the following relation holds

$$
\begin{aligned}
& (1-p) u(w+p \pi)+p u(w-(1-p) \pi) \\
& \quad \leq u((1-p)(w+p \pi)+p(w-(1-p) \pi))=u(w)
\end{aligned}
$$

Thus, since $\bar{b}^{I}>(1-p) \pi$ and $\bar{b}^{E} \leq(1-p) \pi$ it must be the case that $\bar{b}^{I}>\bar{b}^{E}$, which together with condition A.12 implies that $E^{E}(b) \leq E^{I}(b)$.

Proof of Proposition 10. To prove this result, we examine the relation between the equilibrium cdf in the exclusion and inclusion error scenarios with loss averse bidders. In particular, $F^{E}(b) \geq F^{I}(b)$ if, and only if

$$
\lambda u(b) \geq(1-p) \lambda u(b)+p[u(\pi)-u(\pi-b)]
$$

The last inequality holds true both with linear and concave $u(\cdot)$. Notice that when $u(\cdot)$ is linear, $u(b)=u(\pi)-u(\pi-b)$. Thus, for any $\lambda \geq 1$, such that $\lambda u(b) \geq u(b)$, the inequality holds true. With concave $u(\cdot)$, it holds that $\lambda u(b) \geq$ $u(b) \geq u(\pi)-u(\pi-b)$, whereby the last relation is satisfied due to diminishing marginal utility (with strict inequality for strict concavity). ${ }^{28}$

We need to show now that $\bar{b}^{E}<\bar{b}^{I}$. Notice that under loss aversion $\bar{b}^{E}$ solves

$$
(1-p) u\left(\pi-\bar{b}^{E}\right)=\lambda p u\left(\bar{b}^{E}\right)
$$

while $\bar{b}^{I}$ solves

$$
u\left(\pi-\bar{b}^{I}\right)=p u(\pi)
$$

If we evaluate condition (A.15) in $\bar{b}^{I}$ it must be the case that

$$
(1-p) u\left(\pi-\bar{b}^{I}\right)<\lambda p u\left(\bar{b}^{I}\right)
$$

\footnotetext{
${ }^{28}$ Notice that when $u(\cdot)$ is convex the relation between average bids under exclusion and inclusion errors depends both on $\lambda$ and on the curvature of $u(\cdot)$.
} 
Notice that due to condition (A.16) and to concavity the last condition becomes

$$
(1-p) u\left(\pi-\bar{b}^{I}\right)=(1-p) p u(\pi) \leq p u((1-p) \pi)<\lambda p u\left(\bar{b}^{I}\right) .
$$

To show that the last relation holds true it is enough to show that $\bar{b}^{I}>(1-p) \pi$ by arguing that it cannot be otherwise. Indeed $(1-p) \pi$ does not satisfy condition (A.16). Moreover, due to concavity $\lambda u(\pi-(1-p) \pi=\lambda u(p \pi)>u(p \pi)>p u(\pi)$. Thus, $\bar{b}^{E}<\bar{b}^{I}$. This result together with $F^{E}(b) \geq F^{I}(b)$ implies that $E^{E}(b)<E^{I}(b)$.

Proof of Proposition 11. We need to show that

$$
\int_{0}^{\bar{b}^{I}}\left[1-G^{I}(b)\right] d b \leq \int_{0}^{\bar{b}^{E}}\left[1-G^{E}(b)\right] d b
$$

that is, the average equilibrium bid under inclusion errors is smaller or equal to the average equilibrium bid under exclusion errors.

It is immediate to show that $G^{I}(b) \geq G^{E}(b)$ for every $b$. Indeed, the following relation

$$
\begin{aligned}
& G^{I}(b)=\frac{(1-p)[u(\pi+b)-u(\pi)]+p u(b)}{(1-p)[u(\pi+b)-u(b)]} \geq \\
& \frac{u(\pi+b)-u(\pi)}{(1-p)[u(\pi+b)-u(b)]}=G^{E}(b)
\end{aligned}
$$

holds true, since when $u(x)$ is linear

$$
(1-p)[u(\pi+b)-u(\pi)]+p u(b)=u(\pi+b)-u(\pi)
$$

while when $u(x)$ is concave

$$
u(b)>u(\pi+b)-u(\pi)
$$

that implies

$$
(1-p)[u(\pi+b)-u(\pi)]+p u(b)>u(\pi+b)-u(\pi) .
$$

To complete the proof, we also need to show that $\bar{b}^{I} \leq \bar{b}^{E}$. It is immediate to show that the two upper bounds coincide under linearity of $u(\cdot)$. Suppose that $u(\cdot)$ is concave. We show that $\bar{b}^{I}<\bar{b}^{E}$. Notice that under loss aversion $\bar{b}^{E}$ solves

$$
(1-p) u\left(\bar{b}^{E}\right)+p u\left(\pi+\bar{b}^{E}\right)=u(\pi)
$$

while $\bar{b}^{I}$ solves

$$
u\left(\bar{b}^{I}\right)=(1-p) u(\pi) .
$$

We can show that condition (A.19) evaluated in $\bar{b}^{I}$ that solves (A.20) delivers

$$
(1-p) u\left(\bar{b}^{I}\right)+p u\left(\pi+\bar{b}^{I}\right)=(1-p)^{2} u(\pi)+p u\left(\pi+\bar{b}^{I}\right)>u(\pi)
$$


as $p>\frac{1}{2}$ by assumption. Thus, it must be the case that $\bar{b}^{I}<\bar{b}^{E}$. This result together with condition (A.18) implies that condition (A.17) holds true. Thus, $E^{E}(b) \geq E^{I}(b)$ that holds with equality with linear $u(\cdot)$.

\section{Appendix B: Experimental Instructions}

\section{Instructions for the Prize framing}

[translated from Italian]

Welcome and thank you for participating in this experiment. By closely following our instructions you will have the chance to earn an amount of money that you will receive in cash at the end of the experiment. You are not allowed to talk or communicate with other participants. If you have any questions, raise your hand and one of the assistants will come and give you an answer. The following rules apply to all participants.

\section{General instructions}

- The experiment consists of 40 periods.

- In each period:

- You will be assigned to a group of $\mathbf{2}$ participants, randomly and anonymously paired, and, given your choices and the choices of the other participant, you will have the chance to earn an amount in ECU (100 ECU $=1$ EURO).

- Every participant has a monetary endowment of 1000 ECU (10 euro) and will participate in a contest where, without observing the choices of the other participant, he/she has to decide how many tickets to buy from a minimum of 0 to a maximum of 1000 , at the cost of 1 ECU per ticket.

- The participant who buys most tickets wins the contest and obtains a prize of 1000 ECU (10 euro) given some rules that are illustrated below.

- Earnings are determined in the following way: PROFIT $=$ ENDOWMENT - TICKET EXPENDITURE + PRIZE (IF ANY)

Notice that the ticket expenditure will be subtracted to your final earnings independently of whether you obtain the prize or not

- At the end of the experiment the computer will randomly draw one period.

- Earnings obtained in the selected period, plus 2 euro for filling a questionnaire at the end of the experiment, will be paid in cash. 


\section{Periods 1 - 10}

- The participant in the pair who buys most tickets wins the contest (in case of a tie, the winner is randomly drawn by the computer with probability $\frac{1}{2}$ ).

- The winner obtains the prize, the loser does not obtain the prize.

\section{Example}

You buy a tickets and the other participant buys $\mathbf{b}$, where $\mathbf{a}>\mathbf{b}$. Hence, you won the contest. Therefore:

- you obtain the prize and earn:

1000 (endowment)

- a (tickets expenditure)

+1000 (prize)

$=2000-\mathbf{a}($ period earnings in ECU)

- the other participant does not obtain the prize and earns:

1000 (endowment)

$-\mathbf{b}$ (tickets expenditure)

$=1000-\mathbf{b}($ period earnings in ECU)

\section{Periods 11 - 20}

- The participant in the pair who buys most tickets wins the contest (in case of a tie, the winner is randomly drawn by the computer with probability $\frac{1}{2}$ ).

- An error in the prize assignment may occur: with probability $\frac{1}{4}$ the winner does not obtain the prize. Thus:

- if no error occurs (with probability $\frac{3}{4}$ ), the winner obtain the prize and the loser does not obtain it.

- if the error occurs (with probability $\frac{1}{4}$ ), the winner does not obtain the prize and the loser does not obtain the prize (that is none obtains the prize).

- The computer will determine whether the error occurs depending on the realization of two coin tosses that will be shown on the sceen as illustrated in the figure. If the realization is (head, head), an error occurs; if the realization is not (head, head), no error occurs. 


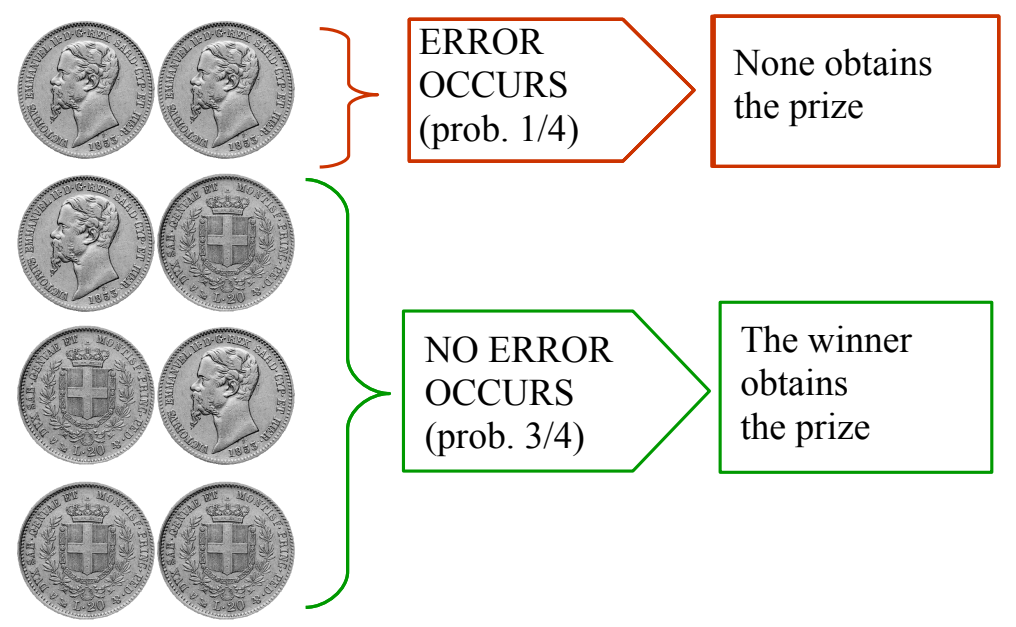

Example: You buy a tickets and the other participant buys $\mathbf{b}$, where $\mathbf{a}>\mathbf{b}$. Hence, you won the contest.

- If no error occurs you obtain the prize and earn:

1000 (endowment)

-a (tickets expenditure)

$+1000($ prize $)$
$=2000-\mathbf{a}($ period earnings in ECU)

- If an error occurs you do not receive the prize and earn:

1000 (endowment)

-a (ticket expenditures)

$=1000-\mathbf{a}($ period earnings in ECU)

- Whatever the realization of the coin tosses, the other participant, who lost the contest, does not obtain the prize and earns:

1000 (endowment)

-b (tickets expenditure)

$=1000-\mathbf{b}$ (periods earnings in ECU)

\section{Periods 21 - 30}

- The participant in the pair who buys most tickets wins the contest (in case of a tie, the winner is randomly drawn by the computer with probability $\frac{1}{2}$ ).

- An error in the prize assignment may occur: with probability $\frac{1}{4}$ the loser obtains the prize. Thus:

- if no error occurs (with probability $\left.\frac{3}{4}\right)$ ), the winner obtains the prize and the loser does not obtain the prize. 
- if an error occurs (with probability $\frac{1}{4}$ ), the winner obtains the prize and chi the loser obtains the prize (that is both obtain the prize).

- The computer will determine whether the error occurs depending on the realization of two coin tosses that will be shown on the sceen as illustrated in the figure. If the realization is (head, head), an error occurs; if the realization is not (head, head), no error occurs.

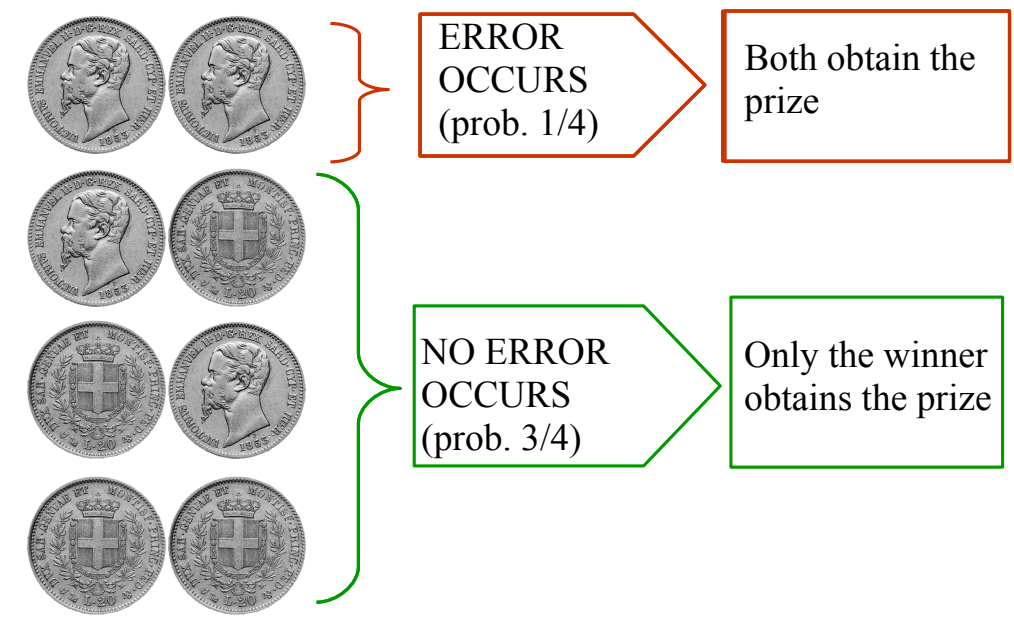

Example: You buy a tickets and the other participant buys $\mathbf{b}$, where $\mathbf{a}>\mathbf{b}$. Hence, you won the contest.

- Whatever the realization of the coin tosses, you obtain the prize and earni:

1000 (endowment)

$-\mathbf{a}$ (tickets expenditure)

+1000 (prize)

$=2000-\mathbf{a}($ period earnings in ECU)

- If no error occurs the other participant, who lost the contest, does not obtain the prize and earns:

1000 (endowment

- b (tickets expenditure)

$=1000-\mathbf{b}$ (periods earnings in ECU)

- If an error occurs also the other participants obtains the prize and earns:

1000 (endowment)

-b (tickets expenditure)

+1000 (prize)

$=2000-\mathbf{b}($ period earnings in ECU) 


\section{Periods $31-40$}

- The participant in the pair who buys most tickets wins the contest (in case of a tie, the winner is randomly drawn by the computer with probability $\frac{1}{2}$ ).

- An error in the prize assignment may occur: with probability $\frac{1}{4}$ the winner does not obtain the prize and the loser obtains the prize. Thus:

- if no error occurs (with probability $\frac{3}{4}$ ), the winner obtains the prize and the loser does not obtain the prize.

- if an error occurs (with probability $\frac{1}{4}$ ), the winner does not obtain the prize e the loser obtains the prize.

- The computer will determine whether the error occurs depending on the realization of two coin tosses that will be shown on the sceen as illustrated in the figure. If the realization is (head, head), an error occurs; if the realization is not (head, head), no error occurs.

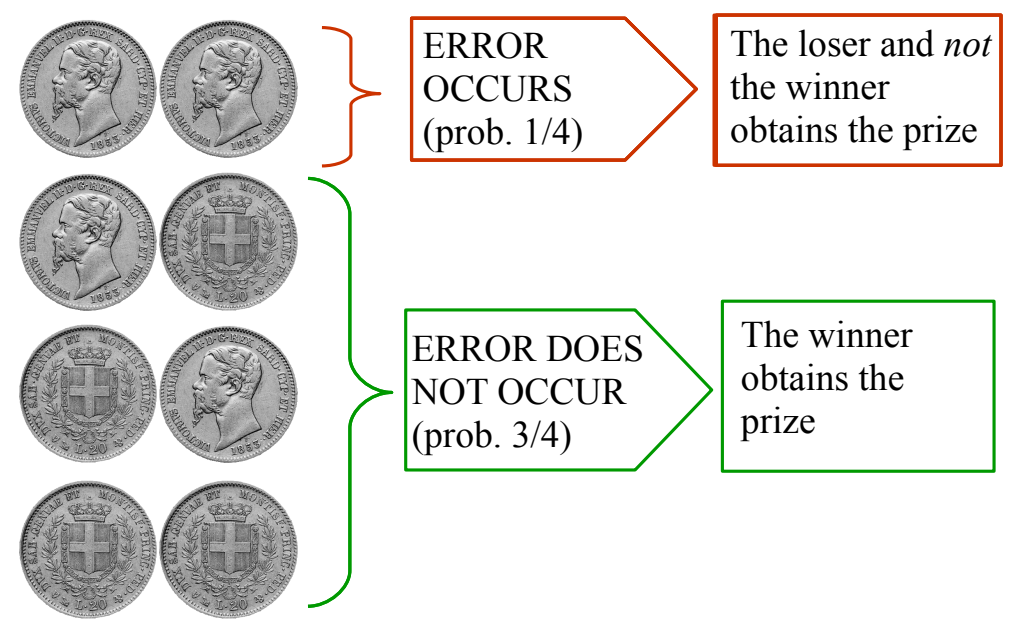

Example: You buy a tickets and the other participant buys $\mathbf{b}$, where $\mathbf{a}>\mathbf{b}$. Hence, you won the contest. If no error occurs:

- you obtain the prize and earn:

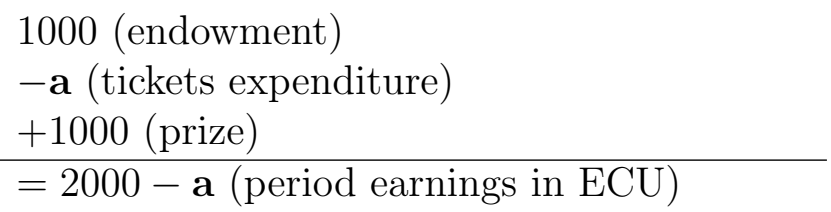

- the other participant does not obtain the prize and earns:

1000 (endowment)

-b (tickets expenditure)

$=1000-\mathbf{b}($ period earnings in ECU $)$

If an error occurs: 
- you do not obtain the prize and earn:

1000 (endowment)
$-\mathbf{a}($ tickets expenditure $)$
$=1000-\mathbf{a}$ (period earnings in ECU)

- the other participant obtains the prize and earns:

1000 (endowment)
$-\mathbf{b}$ (tickets expenditure)
+1000 (prize)
$=2000-\mathbf{b}$ (period earnings in ECU)

\section{Instructions for the Penalty framing}

[translated from Italian]

Welcome and thank you for participating in this experiment. By closely following our instructions you will have the chance to earn an amount of money that you will receive in cash at the end of the experiment. You are not allowed to talk or communicate with other participants. If you have any questions, raise your hand and one of the assistants will come and give you an answer. The following rules apply to all participants.

\section{General instructions}

- The experiment consists of 40 periods.

- In each period:

- You will be assigned to a group of $\mathbf{2}$ participants, randomly and anonymously paired, and, given your choices and the choices of the other participant, you will have the chance to earn an amount in ECU (100 ECU $=1$ EURO).

- Every participant has a monetary endowment of 2000 ECU (20 euro) and participates in a contest where, without observing the choices of the other participant, he/she has to decide how many tickets to buy from a minimum of 0 to a maximum of 1000, at the cost of 1 ECU per ticket.

- The participant who buys most tickets wins the contest and obtains a prize of 1000 ECU (10 euro) given some rules that are illustrated below.

- The participant who buys least tickets loses the contest and obtains a penaly of Chi 1000 ECU (10 euro) given some rules that are illustrated below.

- Earnings are determined in the following way: PROFIT $=$ ENDOWMENT - TICKETS EXPENDITURE - PENALTY (IF ANY)

Notice that the ticket expenditure will be subtracted to your final earnings independently of whether you obtain the penalty or not. 
- At the end of the experiment the computer will randomly draw one period.

- Earnings obtained in the selected period, plus 2 euro for filling a questionnaire at the end of the experiment, will be paid in cash.

\section{Periods 1 - 10}

- The participant who buys least tickets loses the contest (in case of a tie, the loser is randomly drawn by the computer with probability $\frac{1}{2}$ ).

- The loser obtains a penalty, the winner does not obtain a penalty.

\section{Example}

You buy a tickets and the other participant buys $\mathbf{b}$, where $\mathbf{a}>\mathbf{b}$. Hence, you won the contest. Therefore:

- you do not obtain the penalty and earn:

2000 (endowment)

-a (tickets expenditure)

$=2000-\mathbf{a}($ period earnings in ECU)

- the other participant obtains the penalty and earns:

2000 (endowment)

-b (tickets expenditure)

-1000 (penalty)

$=1000-\mathbf{b}($ period earnings in ECU)

\section{Periods $11-20$}

- The participant who buys least tickets loses the contest (in case of a tie, the loser is randomly drawn by the computer with probability $\frac{1}{2}$ ).

- An error in the penalty assignment may occur: with probability $\frac{1}{4}$ the winner obtains the penalty. Thus:

- if no error occurs (with probability $\frac{3}{4}$ ), the loser obtains the penalty and the winner does not obtain the penalty.

- if an error occurs (with probability $\frac{1}{4}$ ), the loser obtains the penalty and the winner obtains the penalty (that is both obtain the penalty).

- The computer will determine whether the error occurs depending on the realization of two coin tosses that will be shown on the sceen as illustrated in the figure. If the realization is (head, head), an error occurs; if the realization is not (head, head), no error occurs. 


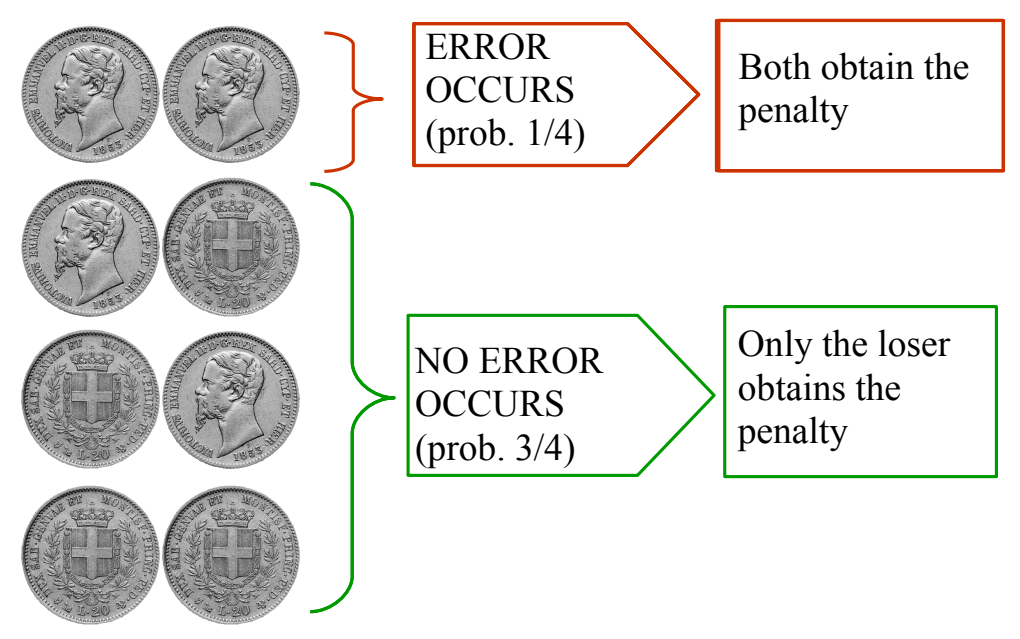

\section{Example}

You buy a tickets and the other participant buys $\mathbf{b}$, where $\mathbf{a}>\mathbf{b}$. Hence, you won the contest. Therefore:

- If no error occurs you do not obtain the penalty and earn:

2000 (endowment)

$\frac{-\mathbf{a} \text { (tickets expenditure) }}{=2000-\mathbf{a} \text { (period earnings in ECU) }}$

- If an error occurs you obtain the penalty and earn:

2000 (endowment)

-a (tickets expenditure)

-1000 (penalty)

$=1000-\mathbf{a}($ period earnings in ECU)

- Independently of the realization of the coin tosses, the other participant, who lost the contest, obtains the penalty and earns:

2000 (endowment)

-b (tickets expenditure)

-1000 (penalty)

$=1000-\mathbf{b}($ period earnings in ECU)

\section{Periods $21-30$}

- The participant who buys least tickets loses the contest (in case of a tie, the loser is randomly drawn by the computer with probability $\frac{1}{2}$ ).

- An error in the penalty assignment may occur: with probability $\frac{1}{4}$ the loser does not obtain the penalty. Thus:

- if no error occurs (with probability $\frac{3}{4}$ ), the loser obtains the penalty and the winner does not obtain the penalty. 
- if an error occurs (with probability $\frac{1}{4}$ ), the loser does not obtain the penalty and the winner does not obtain the penalty (that is none obtains the penalty).

- The computer will determine whether the error occurs depending on the realization of two coin tosses that will be shown on the sceen as illustrated in the figure. If the realization is (head, head), an error occurs; if the realization is not (head, head), no error occurs.

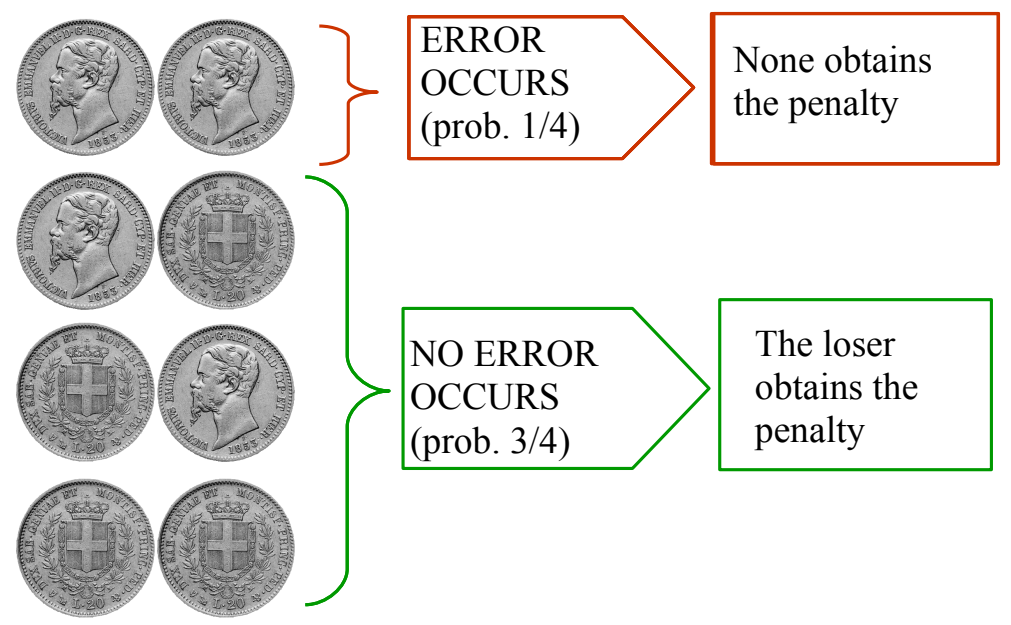

\section{Example}

You buy a tickets and the other participant buys $\mathbf{b}$, where $\mathbf{a}>\mathbf{b}$. Hence, you won the contest. Therefore:

- Independently of the realization of the coin tosses, you do not obtain the penalty and earn:

2000 (endowment)

-a (tickets expenditure)

$=2000-\mathbf{a}($ period earnings in ECU)

- If no error occurs, the other participant, who lost the contest, obtains the penalty and earns:

2000 (endowment)

-b (tickets expenditure)

- 1000 (penalty)

$=1000-\mathbf{b}($ period earnings in ECU $)$

- If an error occurs, also the other participant does not obtain the penalty and earns:

2000 (endowment)

-b (tickets expenditure)

$=2000-\mathbf{b}$ (period earnings in ECU) 


\section{Periods $31-40$}

- The participant who buys least tickets loses the contest (in case of a tie, the loser is randomly drawn by the computer with probability $\frac{1}{2}$ ).

- An error in the penalty assignment may occur: with probability $\frac{1}{4}$ the loser does not obtain the penalty and the winner obtains the penalty. Thus:

- if no error occurs (with probability $\frac{3}{4}$ ), the loser obtains the penalty and the winner does not obtain the penalty.

- if an error occurs (with probability $\frac{1}{4}$ ), the loser does not obtain the penalty and the winner obtains the penalty.

- The computer will determine whether the error occurs depending on the realization of two coin tosses that will be shown on the sceen as illustrated in the figure. If the realization is (head, head), an error occurs; if the realization is not (head, head), no error occurs.

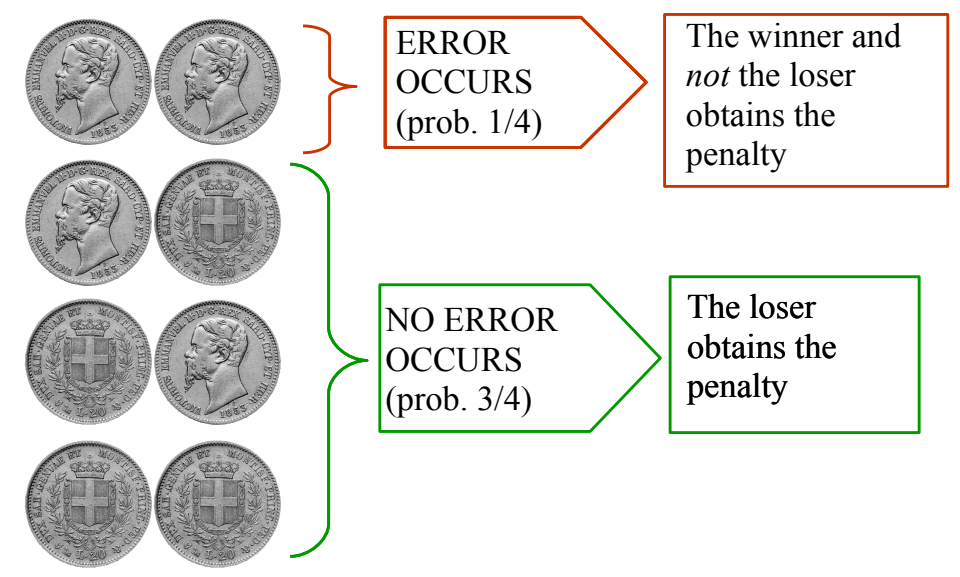

You buy a tickets and the other participant buys $\mathbf{b}$, where $\mathbf{a}>\mathbf{b}$. Hence, you won the contest. If no error occurs:

- you do not obtain the penalty and earn:

2000 (endowment)

- a (tickets expenditure)

$=2000-\mathbf{a}($ period earnings in ECU)

- the other participant obtains the penalty and earns:

2000 (endowment)

-b (tickets expenditure)

-1000 (penalty)
$=1000-\mathbf{b}($ period earnings in ECU)

If an error occurs: 
- you obtain the penalty and ear:

2000 (endowment)

-a (tickets expenditure)

-1000 (penalty)

$=1000-\mathbf{a}($ period earnings in ECU)

- the other participant does not obtain the penalty and earns:

2000 (endowment)

- b (tickets expenditure)

$=2000-\mathbf{b}$ (period earnings in ECU) 